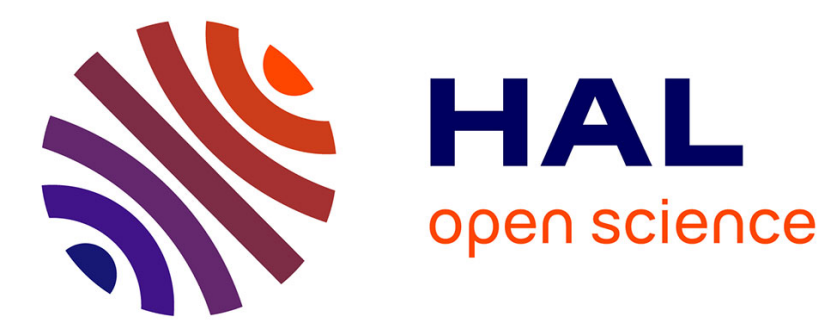

\title{
Controllability of a one-dimensional fractional heat equation: theoretical and numerical aspects
}

\author{
Umberto Biccari, Víctor Hernández-Santamaría
}

\section{To cite this version:}

Umberto Biccari, Víctor Hernández-Santamaría. Controllability of a one-dimensional fractional heat equation: theoretical and numerical aspects. 2017. hal-01562358v2

\section{HAL Id: hal-01562358 \\ https://hal.science/hal-01562358v2}

Preprint submitted on 29 Nov 2017

HAL is a multi-disciplinary open access archive for the deposit and dissemination of scientific research documents, whether they are published or not. The documents may come from teaching and research institutions in France or abroad, or from public or private research centers.
L'archive ouverte pluridisciplinaire HAL, est destinée au dépôt et à la diffusion de documents scientifiques de niveau recherche, publiés ou non, émanant des établissements d'enseignement et de recherche français ou étrangers, des laboratoires publics ou privés. 


\title{
Controllability of a one-dimensional fractional heat equation: theoretical and numerical aspects
}

\author{
Umberto Biccari $^{* \dagger} \quad$ Víctor Hernández-Santamaría $* \dagger$
}

\begin{abstract}
We analyze the controllability problem for a one-dimensional heat equation involving the fractional Laplacian $\left(-d_{x}^{2}\right)^{s}$ on the interval $(-1,1)$. Using classical results and techniques, we show that, acting from an open subset $\omega \subset(-1,1)$, the problem is null-controllable for $s>1 / 2$ and that for $s \leq 1 / 2$ we only have approximate controllability. Moreover, we deal with the numerical computation of the control employing the penalized Hilbert Uniqueness Method (HUM) and a finite element (FE) scheme for the approximation of the solution to the corresponding elliptic equation. We present several experiments confirming the expected controllability properties.
\end{abstract}

\section{Introduction and main results}

Let $\omega \subset(-1,1)$ be an open and nonempty subset. In this work, we consider the following nonlocal one-dimensional heat equation defined on the domain $(-1,1) \times(0, T)$

$$
\begin{cases}z_{t}+\left(-d_{x}^{2}\right)^{s} z=g \mathbf{1}_{\omega}, & (x, t) \in(-1,1) \times(0, T) \\ z=0, & (x, t) \in[\mathbb{R} \backslash(-1,1)] \times(0, T) \\ z(x, 0)=z_{0}(x), & x \in(-1,1),\end{cases}
$$

where $z_{0} \in L^{2}(-1,1)$ is a given initial datum. In $(1.1)$, for all $s \in(0,1),\left(-d_{x}^{2}\right)^{s}$ denotes the one-dimensional fractional Laplace operator, which is defined as the following singular integral

$$
\left(-d_{x}^{2}\right)^{s} u(x)=c_{1, s} \text { P.V. } \int_{\mathbb{R}} \frac{u(x)-u(y)}{|x-y|^{1+2 s}} d y .
$$

Here, $c_{1, s}$ is a normalization constant given by

$$
c_{1, s}=\frac{s 2^{2 s} \Gamma\left(\frac{1+2 s}{2}\right)}{\sqrt{\pi} \Gamma(1-s)},
$$

where $\Gamma$ is the usual Gamma function. Moreover, we have to mention that, for having a completely rigorous definition of the fractional Laplace operator, it is necessary to introduce also the class of functions $u$ for which computing $\left(-d_{x}^{2}\right)^{s} u$ makes sense. We postpone this discussion to the next section.

The analysis of non-local operators and non-local PDEs is a topic in continuous development. A motivation for this growing interest relies in the large number of possible applications in the modeling of several complex phenomena for which a local approach turns up to be inappropriate or limiting. Indeed, there is an ample spectrum of situations in which a non-local equation gives a significantly better description than a PDE of the problem one wants to analyze. Among others, we mention applications in turbulence ([5]), anomalous transport and diffusion ([8], 37]), elasticity ([19]), image processing ([27]), porous media flow ([48]), wave propagation in heterogeneous high contrast media

\footnotetext{
${ }^{*}$ DeustoTech, University of Deusto, 48007 Bilbao, Basque Country, Spain.

${ }^{\dagger}$ Facultad Ingeniería, Universidad de Deusto, Avda Universidades 24, 48007 Bilbao, Basque Country, Spain. Emails: umberto.biccari@deusto.es, u.biccari@gmail.com, victor.santamaria@deusto.es
} 
([49]). Also, it is well known that the fractional Laplacian is the generator of s-stable processes, and it is often used in stochastic models with applications, for instance, in mathematical finance ([35, 42]).

One of the main differences between these non-local models and classical Partial Differential Equations is that the fulfillment of a non-local equation at a point involves the values of the function far away from that point.

In the present paper, we are interested in studying control properties for equation (1.1). In more detail, we aim to give an answer to the following question:

given any $T>0$ and any initial datum $z_{0} \in L^{2}(-1,1)$, can we find a control function $g \in L^{2}(\omega \times(0, T))$

such that the corresponding solution to $(1.1)$ satisfies $z(x, T)=0$ ?

It is well-known that the classical local heat equation (as well as many other general variants) is null-controllable for any time $T>0$ (see, e.g., [21, 24, 33]). Nevertheless, to the best of our knowledge, there are few results in the literature on the null-controllability of the fractional heat equation, and none of them is for a problem involving the fractional Laplacian in its integral form (1.2). The existing results ([39,40]), instead, deal with the so-called spectral fractional Laplace operator, whose definition will be given later.

In this paper, we deal with the controllability of [1.1, both from the theoretical and the numerical point of view. Employing spectral analysis techniques based on the works [31, 32], the first main result that we obtain is the following

Theorem 1.1. Given any $z_{0} \in L^{2}(-1,1)$, the parabolic problem (1.1) is null-controllable at time $T>0$ with a control function $g \in L^{2}(\omega \times(0, T))$ if and only if $s>1 / 2$.

Furthermore, even if for $s \leq 1 / 2$ null controllability for (1.1) fails, we still have the following result of approximate controllability, as a consequence of unique continuation properties for the fractional Laplace operator ([20]).

Theorem 1.2. Let $s \in(0,1)$. Given any $z_{0} \in L^{2}(-1,1)$, there exists a control function $g \in L^{2}(\omega \times(0, T))$ such that the unique solution $z$ to the parabolic problem (1.1) is approximately controllable at time $T>0$.

Theorems 1.1 and 1.2 will then find a confirmation in the study of the corresponding numerical control problem. For this purpose, we will employ the penalized Hilbert Uniqueness Method, which relies in the classical works of Glowinski and Lions ([28, 29]). This method is very general and it can be applied to a broad class of PDE control problems, see, for instance, [12, 13, 15, 30]. However, all of the previous works have one thing in common: they are devoted to the study of equations of local nature. Here, we will see that, when dealing with a nonlocal equation as (1.1), new issues arise during the numerical implementation.

In this context, for the resolution of the numerical control problem, we will need a finite element (FE) approximation of the solution to the following non-local Poisson equation

$$
\begin{cases}\left(-d_{x}^{2}\right)^{s} u=f, & x \in(-1,1) \\ u \equiv 0, & x \in \mathbb{R} \backslash(-1,1) .\end{cases}
$$

In the recent past, the fractional Laplacian has been widely analyzed also from the point of view of numerical analysis. We refer, for instance, to the works [2, 3, 11]. There, the authors present a FE scheme for implementing the solution of 1.3$)$ in a bounded domain $\Omega \subset \mathbb{R}^{2}$. In particular, they provide appropriate quadrature rules in order to solve numerically the variational formulation associated to the problem. Moreover, in [3, 11] it is also developed an accurate analysis of the efficiency of the FE method, employing several existing results. The techniques of the aforementioned works have then been applied in [1], combined with a convolution quadrature approach, for solving evolution equations involving the fractional Laplacian. For the sake of completeness, we also mention [10], where it is presented a discretization of the spectral fractional Laplacian and its application to the evolutionary case [9], and [41], where the same problem is treated applying the well known extension of Caffarelli and Silvestre ([16]).

In the present paper, we propose a FE approximation for the fractional Poisson equation (1.3) which does not require any quadrature rule. Indeed, exploiting the one-dimensional nature of the problem, each entry of the stiffness matrix can be computed explicitly in terms of its position, the parameter $s$ and the mesh size. This, in particular, allows a quick and simple implementation of the control problem.

This paper is organized as follows. In Section 2 , we briefly present the functional setting and some existing theory related to the problems that we are going to analyze. In particular, we give a more accurate definition of the fractional 
Laplace operator, we discuss the known results on the controllability of fractional parabolic equations and we introduce the variational formulation associated to (1.3) (needed for the development of the FE scheme). Section 3 is devoted to the proof of Theorems 1.1 and 1.2. In Section 4, we describe the penalized HUM with its application to our control problem. Moreover, we present our FE method for the elliptic equation (1.3), that then will be employed for the numerical control of (1.1). In Section 5 we present and comment the results of our numerical simulations. Finally, in Appendix A we include the complete details for computing the stiffness matrix associated to our FE scheme.

\section{Preliminary results on the fractional Laplace operator}

In this Section, we introduce some preliminary result that will be useful in the remainder of the paper.

We start by giving a more rigorous definition of the fractional Laplace operator, as we have anticipated in Section 1 Let

$$
\mathcal{L}_{s}^{1}(\mathbb{R}):=\left\{u: \mathbb{R} \longrightarrow \mathbb{R}: u \text { measurable }, \int_{\mathbb{R}} \frac{|u(x)|}{(1+|x|)^{1+2 s}} d x<\infty\right\}
$$

For any $u \in \mathcal{L}_{s}^{1}$ and $\varepsilon>0$ we set

$$
\left(-d_{x}^{2}\right)_{\varepsilon}^{s} u(x)=c_{1, s} \int_{|x-y|>\varepsilon} \frac{u(x)-u(y)}{|x-y|^{1+2 s}} d y, \quad x \in \mathbb{R} .
$$

The fractional Laplacian is then defined by the following singular integral

$$
\left(-d_{x}^{2}\right)^{s} u(x)=c_{1, s} P . V . \int_{\mathbb{R}} \frac{u(x)-u(y)}{|x-y|^{1+2 s}} d y=\lim _{\varepsilon \rightarrow 0^{+}}\left(-d_{x}^{2}\right)_{\varepsilon}^{s} u(x), \quad x \in \mathbb{R},
$$

provided that the limit exists.

We notice that if $0<s<1 / 2$ and $u$ is a smooth function, for example bounded and Lipschitz continuous on $\mathbb{R}$, then the integral in $\left[2.1\right.$ is in fact not really singular near $x$ (see e.g. [18, Remark 3.1]). Moreover, $\mathcal{L}_{s}^{1}(\mathbb{R})$ is the right space for which $v:=\left(-d_{x}^{2}\right)_{\varepsilon}^{s} u$ exists for every $\varepsilon>0, v$ being also continuous at the continuity points of $u$.

It is by now well-known (see, e.g., [18]) that the natural functional setting for problems involving the Fractional Laplacian is the one of the fractional Sobolev spaces. Since these spaces are not as familiar as the classical integral order ones, for the sake of completeness, we recall here their definition.

Given $s \in(0,1)$, the fractional Sobolev space $H^{s}(-1,1)$ is defined as

$$
H^{s}(-1,1):=\left\{u \in L^{2}(-1,1): \frac{|u(x)-u(y)|}{|x-y|^{\frac{1}{2}+s}} \in L^{2}((-1,1) \times(-1,1))\right\} .
$$

It is classical that this is a Hilbert space, endowed with the norm (derived from the scalar product)

$$
\|u\|_{H^{s}(-1,1)}:=\left[\|u\|_{L^{2}(-1,1)}^{2}+|u|_{H^{s}(-1,1)}^{2}\right]^{\frac{1}{2}}
$$

where the term

$$
|u|_{H^{s}(-1,1)}:=\left(\int_{-1}^{1} \int_{-1}^{1} \frac{|u(x)-u(y)|^{2}}{|x-y|^{1+2 s}} d x d y\right)^{\frac{1}{2}}
$$

is the so-called Gagliardo seminorm of $u$. We set

$$
H_{0}^{s}(-1,1):={\overline{C_{0}^{\infty}(-1,1)}}^{H^{s}(-1,1)}
$$

the closure of the continuous infinitely differentiable functions with compact support in $(-1,1)$ with respect to the $H^{s}(-1,1)$-norm. The following facts are well-known. 
- For $0<s \leq \frac{1}{2}$, the identity $H_{0}^{s}(-1,1)=H^{s}(-1,1)$ holds. This is because, in this case, the $C_{0}^{\infty}(-1,1)$ functions are dense in $H^{s}(-1,1)$ (see, e.g., [36, Theorem 11.1]).

- For $\frac{1}{2}<s<1$, we have $H_{0}^{s}(-1,1)=\left\{u \in H^{s}(\mathbb{R}): u=0\right.$ in $\left.\mathbb{R} \backslash(-1,1)\right\}$ ([23]).

Finally, in what follows we will indicate with $H^{-s}(-1,1)=\left(H^{s}(-1,1)\right)^{\prime}$ the dual space of $H^{s}(-1,1)$ with respect to the pivot space $L^{2}(-1,1)$.

A more exhaustive description of fractional Sobolev spaces and of their properties can be found in several classical references (see, e.g., [4, 18, 36]).

Let us now discuss the parabolic equation (1.1). First of all, we mention that the issues of existence, uniqueness and regularity of the solutions have been studied by several authors. Among others, we mention the works [7, 22, 34]. In particular, in [34, Theorem 26] it is showed that, assuming $z_{0} \in L^{2}(\Omega)$ and $g \in L^{2}\left(0, T ; H^{-s}(\Omega)\right)$, then equation (1.1) admits a unique weak solution $z \in L^{2}\left(0, T ; H_{0}^{s}(\Omega)\right) \cap C\left([0, T] ; L^{2}(\Omega)\right)$ with $z_{t} \in L^{2}\left(0, T ; H^{-s}(\Omega)\right)$. Notice that taking as in our case $g \in L^{2}(\omega \times(0, T))$, the same result holds due to the continuous injection of $L^{2}$ into $H^{-s}$.

In this paper we are mainly interested in the study of control properties for the parabolic system (1.1). For the sake of completeness, we include below the definitions of null and approximate controllability.

Definition 2.1. System (1.1) is said to be null-controllable at time $T>0$ if, for any $z_{0} \in L^{2}(-1,1)$, there exists $g \in L^{2}(\omega \times(0, T))$ such that the corresponding solution z satisfies

$$
z(x, T)=0 .
$$

Definition 2.2. System (1.1) is said to be approximately controllable at time $T>0$ if, for any $z_{0}, z_{T} \in L^{2}(-1,1)$ and any $\delta>0$, there exists $g \in L^{2}(\omega \times(0, T))$ such that the corresponding solution $z$ satisfies

$$
\left\|z(x, T)-z_{T}\right\|_{L^{2}(-1,1)} \leq \delta .
$$

We already mentioned that, to the best of our knowledge, there are no results in the literature concerning the controllability of the fractional heat equation involving the integral operator 2.17. The existing ones deal with the spectral definition of the fractional Laplace operator, which is given as follows.

Let $\left\{\psi_{k}, \lambda_{k}\right\}_{k \in \mathbb{N}} \subset H_{0}^{1}(-1,1) \times \mathbb{R}^{+}$be the set of normalized eigenfunctions and eigenvalues of the Laplace operator in $(-1,1)$ with homogeneous Dirichlet boundary conditions, so that $\left\{\psi_{k}\right\}_{k \in \mathbb{N}}$ is an orthonormal basis of $L^{2}(-1,1)$ and

$$
\left\{\begin{array}{l}
-d_{x}^{2} \psi_{k}=\lambda_{k} \psi_{k}, \\
\psi_{k}(-1)=\psi_{k}(1)=0 .
\end{array} \quad x \in(-1,1),\right.
$$

Then, the spectral fractional Laplacian $\left(-d_{x}^{2}\right)_{S}^{s}$ is defined by

$$
\left(-d_{x}^{2}\right)_{S}^{s} u(x)=\sum_{k \geq 1}\left\langle u, \psi_{k}\right\rangle \lambda_{k}^{s} \psi_{k}(x)
$$

firstly for $u \in C_{0}^{\infty}(-1,1)$ and then for $u \in H_{0}^{s}(-1,1)$ employing a density argument.

It is important to notice that the spectral fractional Laplacian and the fractional Laplacian defined as in 2.1) are two different operators. For instance, definition (2.2) depends on the choice of the domain, while the integral definition does not. For a complete discussion on the differences of these two operators, we refer to [46].

The control problem for the fractional heat equation involving the operator $\left(-d_{x}^{2}\right)_{S}^{s}$ has been analyzed in [39], where the authors proved null controllability provided that $s>1 / 2$. For $s \leq 1 / 2$, instead, null controllability does not hold, not even for $T$ large. This negative result is based on the equivalence (consequence of Müntz Theorem, see, e.g., 45, Page 24]) between the controllability property (more specifically, the possibility of proving an observability inequality), and the following condition for the eigenvalues of the considered operator

$$
\sum_{k \geq 1} \frac{1}{\lambda_{k}}<\infty
$$


which is clearly not satisfied for the spectral fractional Laplacian when $s \leq 1 / 2$, since in that case the eigenvalues are $\lambda_{k}=(k \pi)^{2 s}$. Finally, in [40], the same result as in [39] is obtained in a multi-dimensional setting, by means of a spectral observability condition for a negative self-adjoint operator, which allows to prove the null-controllability of the semi-group that it generates.

As we anticipated in Section 1, the same null controllability result holds for the parabolic equation (1.1). This will be obtained by means of classical tools $([21])$ and by an explicit approximations of the eigenvalues and the eigenfunctions the fractional Laplacian with homogeneous Dirichlet boundary conditions.

We stress that $[1.1]$ is a different model with respect to the ones analyzed in [38, 40], since the operators 2.1$]$ and 2.2. are not equivalent.

We conclude this section by introducing the variational formulation associated to equation $(1.3)$, which is at the heart of our numerical method. That is, find $u \in H_{0}^{s}(-1,1)$ such that

$$
a(u, v)=\int_{-1}^{1} f v d x
$$

for all $v \in H_{0}^{s}(-1,1)$, where the bilinear form $a(\cdot, \cdot): H_{0}^{s}(-1,1) \times H_{0}^{s}(-1,1) \rightarrow \mathbb{R}$ is given by

$$
a(u, v)=\frac{c_{1, s}}{2} \int_{\mathbb{R}} \int_{\mathbb{R}} \frac{(u(x)-u(y))(v(x)-v(y))}{|x-y|^{1+2 s}} d x d y .
$$

Since the bilinear form $a$ is continuous and coercive, Lax-Milgram Theorem immediately implies existence and uniqueness of solutions to the Dirichlet problem (1.3). In more detail, if $f \in H^{-s}(-1,1)$, then (1.3) admits a unique weak solution $u \in H_{0}^{s}(-1,1)$ (see, e.g., [6, Proposition 2.1]). Furthermore, in the literature it is possible to find improved regularity results for the solution to 1.3 , both in Hölder and Sobolev spaces. The interested reader may refer, for instance, to [3, 6, 34, 43, 44].

\section{Proof of the controllability properties}

This section is devoted to study the control properties for the parabolic system (1.1). We begin by proving the null controllability result.

Proof of Theorem 1.1. First of all, for all $\varphi^{T} \in L^{2}(-1,1)$, let $\varphi(x, t)$ be the unique weak solution to the adjoint system

$$
\begin{cases}-\varphi_{t}+\left(-d_{x}^{2}\right)^{s} \varphi=0, & (x, t) \in(-1,1) \times(0, T) \\ \varphi=0, & (x, t) \in[\mathbb{R} \backslash(-1,1)] \times(0, T) \\ \varphi(x, T)=\varphi^{T}(x), & x \in(-1,1) .\end{cases}
$$

Multiplying (1.1) by $\varphi$ and integrating over $(-1,1) \times(0, T)$, it is straightforward to check that $z(x, T)=0$ if and only if

$$
\int_{0}^{T} \int_{-1}^{1} \varphi(x, t) g(x, t) \mathbf{1}_{\omega}(x) d x d t=-\int_{-1}^{1} z_{0}(x) \varphi(x, 0) d x,
$$

In turn, it is classical that 3.2 is equivalent to the existence of a constant $C>0$ such that the following observability inequality holds

$$
\|\varphi(x, 0)\|_{L^{2}(-1,1)}^{2} \leq C \int_{0}^{T}\left|\int_{-1}^{1} \varphi(x, t) g(x, t) \mathbf{1}_{\omega}(x) d x\right|^{2} d t,
$$

Notice that $\varphi$ can be expressed in the basis of the eigenfunctions of the fractional Laplacian on $(-1,1)$ with zero Dirichlet boundary conditions. Namely,

$$
\varphi(x, t)=\sum_{k \geq 1} \varphi_{k} e^{-\lambda_{k}(T-t)} \varrho_{k}(x)
$$


where $\varphi_{k}=\left\langle\varphi^{T}, \varrho_{k}\right\rangle$ and, for $k \geq 1, \varrho_{k}(x)$ are the solutions to the following eigenvalue problem

$$
\begin{cases}\left(-d_{x}^{2}\right)^{s} \varrho_{k}=\lambda_{k} \varrho_{k}, & x \in(-1,1), \quad k \in \mathbb{N} \\ \varrho_{k}=0, & x \in \mathbb{R} \backslash(-1,1) .\end{cases}
$$

Now, plugging (3.4) into (3.3), using the orthonormality of the eigenfunctions $\varrho_{k}$ as a basis of $L^{2}(-1,1)$ and employing the change of variables $T-t \mapsto t$, the observability inequality becomes

$$
\sum_{k \geq 1}\left|\varphi_{k}\right|^{2} e^{-2 \lambda_{k} T} \leq C \int_{0}^{T}\left|\sum_{k \geq 1} \varphi_{k} g_{k}(t) e^{-\lambda_{k} t}\right|^{2} d t
$$

where $g_{k}=\left\langle g \mathbf{1}_{\omega}, \varrho_{k}\right\rangle$.

By means of the classical moment method ([21]), inequalities of the form (3.5] are well known to be true if and only if 2.3 holds and the eigenfunctions $\varrho_{k}$ satisfy the following lower bound:

$$
\left\|\varrho_{k}\right\|_{L^{2}(\omega)} \geq C>0, \forall k \geq 1,
$$

where the constant $C$ is independent of $k$. The proof of [3.6 is an easy adaptation of the one of [32, Lemma 2]. Moreover, according to [31, 32] we have

$$
\lambda_{k}=\left(\frac{k \pi}{2}-\frac{(1-s) \pi}{4}\right)^{2 s}+O\left(\frac{1}{k}\right) .
$$

Therefore, we easily see that the condition 2.3 is satisfied if and only if $s>1 / 2$. If $s \leq 1 / 2$, instead, the series diverges, since it behaves like an harmonic series. In conclusion, the observability inequality 3.3 holds true when $s>1 / 2$, but it is false when $s \leq 1 / 2$. This concludes the proof.

Even if for $s \leq 1 / 2$ null controllability for 1.1 fails, Theorem 1.2 ensures that, for all $s \in(0,1)$, we still have approximate controllability. This is consequence of a unique continuation property for the fractional Laplacian, which has been obtained in [20].

Proof of Theorem 1.2. It is classical (see, e.g., [38, Theorem 5.2]) that the result is true as soon as one has the following unique continuation property for the solution to the adjoint equation (3.1).

Given $s \in(0,1)$ and $\varphi^{T} \in L^{2}(-1,1)$, let $\varphi$ be the unique solution to the system 3.1.

Let $\omega \subset(-1,1)$ be an arbitrary open set. If $\varphi=0$ on $\omega \times(0, T)$, then $\varphi=0$ on

$(-1,1) \times(0, T)$.

Therefore, we are reduced to the proof of the property $(\mathcal{P})$. To this end, let us recall that $\varphi$ can be expressed in the form (3.4) and let us assume that

$$
\varphi=0 \text { in } \omega \times(0, T) .
$$

Let $\left\{\psi_{k_{j}}\right\}_{1 \leq k \leq m_{k}}$ be an orthonormal basis of $\operatorname{ker}\left(\lambda_{k}-\left(-d_{x}^{2}\right)^{s}\right)$. Then, 3.4 can be rewritten as

$$
\varphi(x, t)=\sum_{k \geq 1}\left(\sum_{j=1}^{m_{k}} \varphi_{k_{j}} \psi_{k_{j}}(x)\right) e^{-\lambda_{k}(T-t)}, \quad(x, t) \in(-1,1) \times(-\infty, T) .
$$

Let $z \in \mathbb{C}$ with $\eta:=\mathfrak{R}(z)>0$ and let $N \in \mathbb{N}$. Since the functions $\psi_{k_{j}}, 1 \leq j \leq m_{k}, 1 \leq k \leq N$ are orthonormal, we have that

$$
\left\|\sum_{k=1}^{N}\left(\sum_{j=1}^{m_{k}} \varphi_{k_{j}} \psi_{k_{j}}(x)\right) e^{z(t-T)} e^{-\lambda_{k}(T-t)}\right\|_{L^{2}(-1,1)}^{2} \leq \sum_{k=1}^{N}\left(\sum_{j=1}^{m_{k}}\left|\varphi_{k_{j}}\right|^{2}\right) e^{2 \eta(t-T)} e^{-2 \lambda_{k}(T-t)}
$$




$$
\leq \sum_{k \geq 1}\left(\sum_{j=1}^{m_{k}}\left|\varphi_{k_{j}}\right|^{2}\right) e^{2 \eta(t-T)} e^{-2 \lambda_{k}(T-t)} \leq C e^{2 \eta(t-T)}\left\|\varphi^{T}\right\|_{L^{2}(-1,1)}^{2}
$$

Hence, letting

$$
w_{N}(x, t):=\sum_{k=1}^{N}\left(\sum_{j=1}^{m_{k}} \varphi_{k_{j}} \psi_{k_{j}}(x)\right) e^{z(t-T)} e^{-\lambda_{k}(T-t)},
$$

we have shown that $\left\|w_{N}(x, t)\right\|_{L^{2}(-1,1)} \leq C e^{\eta(t-T)}\left\|\varphi^{T}\right\|_{L^{2}(-1,1)}$. Moreover, we have

$$
\int_{-\infty}^{T} e^{\eta(t-T)}\left\|\varphi^{T}\right\|_{L^{2}(-1,1)} d t=\frac{1}{\eta}\left\|\varphi^{T}\right\|_{L^{2}(-1,1)} \int_{0}^{+\infty} e^{-\tau} d \tau=\frac{1}{\eta}\left\|\varphi^{T}\right\|_{L^{2}(-1,1)} .
$$

Therefore, we can apply the Dominated Convergence Theorem, obtaining

$$
\begin{aligned}
\lim _{N \rightarrow+\infty} \int_{-\infty}^{T} w_{N}(x, t) d t & =\int_{-\infty}^{T} \lim _{N \rightarrow+\infty} w_{N}(x, t) d t=\int_{-\infty}^{T} e^{z(t-T)} \sum_{k \geq 1}\left(\sum_{j=1}^{m_{k}} \varphi_{k_{j}} \psi_{k_{j}}(x)\right) e^{-\lambda_{k}(T-t)} d t \\
& =\sum_{k \geq 1}^{+\infty} \sum_{j=1}^{m_{k}} \varphi_{k_{j}} \psi_{k_{j}}(x) \int_{-\infty}^{T} e^{z(t-T)} e^{-\lambda_{k}(T-t)} d t=\sum_{k \geq 1}^{+\infty} \sum_{j=1}^{m_{k}} \varphi_{k_{j}} \psi_{k_{j}}(x) \int_{0}^{+\infty} e^{-\left(z+\lambda_{k}\right) \tau} d \tau \\
& =\sum_{k \geq 1}^{+\infty} \sum_{j=1}^{m_{k}} \frac{\varphi_{k_{j}}}{z+\lambda_{k}} \psi_{k_{j}}(x), \quad x \in(-1,1), \mathfrak{R}(z)>0 .
\end{aligned}
$$

It follows from (3.7) and (3.8) that

$$
\sum_{k \geq 1}^{+\infty} \sum_{j=1}^{m_{k}} \frac{\varphi_{k_{j}}}{z+\lambda_{k}} \psi_{k_{j}}(x)=0, \quad x \in \omega, \mathfrak{R}(z)>0
$$

This holds for every $z \in \mathbb{C} \backslash\left\{-\lambda_{k}\right\}_{k \in \mathbb{N}}$, using the analytic continuation in $z$. Hence, taking a suitable small circle around $-\lambda_{\ell}$ not including $\left\{-\lambda_{k}\right\}_{k \neq \ell}$ and integrating on that circle we get that

$$
w_{\ell}:=\sum_{j=1}^{m_{\ell}} \varphi_{\ell_{j}} \psi_{\ell_{j}}(x)=0, \quad x \in \omega
$$

According to [20, Theorem 1.4], $\left(-d_{x}^{2}\right)^{s}$ has the unique continuation property in the sense that if $\lambda_{k}$ is an eigenvalue of $\left(-d_{x}^{2}\right)^{s}$ on $(-1,1)$ with Dirichlet boundary conditions, and $\left(\left(-d_{x}^{2}\right)^{s}-\lambda_{k}\right) \varrho_{k}=0$ in $(-1,1)$ with $\varrho_{k}=0$ in $\omega$, then $\varrho_{k}=0$ in $(-1,1)$. This can applied to $w_{\ell}$, in order to conclude $w_{\ell}=0$ in $(-1,1)$ for every $\ell$. Since $\left\{\psi_{\ell_{j}}\right\}_{1 \leq j \leq m_{\ell}}$ are linearly independent in $L^{2}(-1,1)$, we get $\varphi_{\ell_{j}}=0,1 \leq j \leq m_{k}, \ell \in \mathbb{N}$. It follows that $\varphi^{T}=0$ and hence, $\varphi=0$ in $(-1,1) \times(0, T)$, meaning that $\varphi$ enjoys the property (P). As an immediate consequence, we have that our original equation (1.1) is approximately controllable. Our proof is then concluded.

Remark 3.1. According to [20], the elliptic unique continuation property for the fractional Laplacian holds in any space dimension. In view of that, Theorem 1.2 may be extended also to the case $N>1$. On the other hand, the same does not applies to Theorem 1.1. Indeed, the proof of this result use arguments that are designed specifically for one-dimensional problems ([2I]). If one would like to analyze the null-controllability in a general multi-dimensional setting, other tools (for instance Carleman estimates) are needed. As far as we know, these techniques have not been fully developed yet for problems involving the fractional Laplacian on a domain.

Remark 3.2. For the sake of simplicity, in the results presented above we focused on the interval $(-1,1)$. Nevertheless, everything that we did in this section actually holds in the more general case $x \in(-L, L)$ and the extension is immediate. 


\section{The penalized HUM and its implementation}

We devote this Section to the description of the numerical scheme that we are going to employ for solving the control problem. Let us start with a brief description of the so called penalized Hilbert Uniqueness Method (HUM in what follows) that we shall employ for computing the controls for equation (1.1). Here, we will mostly refer to the work of Boyer [12].

Let $(E,\langle\cdot, \cdot\rangle)$ be a Hilbert space whose norm is denoted by $\|\cdot\|$. Let $(A, \mathcal{D}(A))$ be an unbounded operator in $E$ such that $-A$ generates an analytic semi-group in $E$ that we indicate by $t \mapsto e^{-t A}$. Also, we denote $\left(A^{*}, \mathcal{D}\left(A^{*}\right)\right)$ the adjoint of this operator and by $t \mapsto e^{-t A^{*}}$ the corresponding semi-group.

Let $(U,[\cdot, \cdot])$ be another Hilbert space whose norm is denoted by $\mathbb{I} \cdot \rrbracket$. Let $B$ be an unbounded operator from $U$ to $\mathcal{D}\left(A^{*}\right)^{\prime}$ and let $B^{*}: \mathcal{D}\left(A^{*}\right) \rightarrow U$ be its adjoint. Let $T>0$ be given and, for any $y_{0} \in E$ and $v \in L^{2}(0, T$; $U)$, let us consider the non-homogeneous evolution problem

$$
\left\{\begin{array}{l}
y_{t}+A y=B v, \quad t \in[0, T] \\
y(0)=y_{0}
\end{array}\right.
$$

The well posedness of 4.1] is guaranteed by [17, Theorem 2.37]. From now on, we will denote the solution at time $T$ corresponding to the initial datum $y_{0}$ and the control $v$ by

$$
y_{v, y_{0}}(T)=\mathcal{L}_{T}\left(v, y_{0}\right) .
$$

The linear operator $\mathcal{L}_{T}(\cdot, \cdot)$ is then continuous from $L^{2}(0, T ; U) \times E$ into $E$.

In the framework of both controllability notions that we introduced in Section 2 if one control exists it is certainly not unique. In the penalized version of the HUM, we look for a control that is solution to a suitable optimization problem. In particular, for any $\varepsilon>0$, we shall find

$$
v_{\varepsilon}=\min _{v \in L^{2}(0, T ; U)} F_{\varepsilon}(v)
$$

where

$$
F_{\varepsilon}(v):=\frac{1}{2} \int_{0}^{T} \llbracket v(t) \rrbracket^{2} d t+\frac{1}{2 \varepsilon}\left\|\mathcal{L}_{T}\left(v, y_{0}\right)\right\|^{2}, \quad \forall v \in L^{2}(0, T ; U) .
$$

Observe that, for any $\varepsilon>0$, the functional $F_{\varepsilon}$ has a unique minimizer in $L^{2}(0, T ; U)$ that we denote by $v_{\varepsilon}$. This is due to the fact that $F_{\varepsilon}$ is strictly convex, continuous and coercive.

However, the space $L^{2}(0, T ; U)$ in which one has to minimize $F_{\varepsilon}$ is a quite big one and it depends on the time variable. This makes the optimization problem computationally expensive. This issue can be circumvented by considering the following problem, defined on the smaller space $E$. Namely, we consider the minimization problem

$$
q_{\varepsilon}^{T}=\min _{q \in E} J_{\varepsilon}\left(q^{T}\right)
$$

where

$$
J_{\varepsilon}\left(q^{T}\right):=\frac{1}{2} \int_{0}^{T} \llbracket B^{*} e^{-(T-t) A^{*}} q^{T} \rrbracket^{2} d t+\frac{\varepsilon}{2}\left\|q^{T}\right\|^{2}+\left\langle\mathcal{L}_{T}\left(y_{0}, 0\right), q^{T}\right\rangle, \quad \forall q^{T} \in E .
$$

In fact, it is classical to prove that (4.3) and (4.4) are equivalent since, for any $\varepsilon>0$, the minimizers $v_{\varepsilon}$ and $q_{\varepsilon}^{T}$ of the functionals $F_{\varepsilon}$ and $J_{\varepsilon}$, respectively, are related through the formula

$$
v_{\varepsilon}=B^{*} e^{-(T-t) A^{*}} q_{\varepsilon}^{T}, \quad \text { for a.e. } t \in(0, T) .
$$

Notice also that we can express the approximate and null controllability properties of the system, for a given initial datum $y_{0}$, in terms of the behavior of the penalized HUM approach described above. In particular, we have 
Theorem 4.1 (Theorem 1.7 of [12]). Problem (4.1] is approximately controllable from the initial datum $y_{0}$ if and only if we have

$$
\mathcal{L}_{T}\left(v_{\varepsilon}, y_{0}\right)=y_{v_{\varepsilon}, y_{0}}(T) \rightarrow 0, \quad \text { as } \varepsilon \rightarrow 0 .
$$

Problem 4.1] is null-controllable from the initial datum $y_{0}$ if and only if we have

$$
M_{y_{0}}^{2}:=2 \sup _{\varepsilon>0}\left(\inf _{L^{2}(0, T ; U)} F_{\varepsilon}\right)<+\infty
$$

In this case, we have

$$
\begin{array}{r}
\llbracket v_{\varepsilon} \rrbracket_{L^{2}(0, T ; U)} \leq M_{y_{0}}, \\
\left\|\mathcal{L}_{T}\left(v_{\varepsilon}, y_{0}\right)\right\| \leq M_{y_{0}} \sqrt{\varepsilon} .
\end{array}
$$

Observe that the fractional Laplacian $\left(-d_{x}^{2}\right)^{s}$ satisfies the properties required for the operator $A$ (see, e.g., [25, Theorem 2.14]). Therefore, the penalized HUM approach that we just described can be applied to the control problem (1.1). Following the discussion in [12], we expect that, under discretization, the solution $z$ to (1.1) retains the properties of controllability stated in Theorem 4.1. This will be in accordance with the theoretical results obtained in Theorems 1.1 and 1.2 .

To this end, let us study the fully-discrete version of 1.1. For any given mesh $\mathfrak{M}$ and any integer $M>0$, we set $\delta t=T / M$ and we consider an implicit Euler method, with respect to the time variable. More precisely, we consider

$$
\left\{\begin{array}{l}
\mathcal{M}_{h} \frac{z^{n+1}-z^{n}}{\delta t}+\mathcal{A}_{h} z^{n+1}=v_{h}^{n+1} \mathbf{1}_{\omega}, \quad \forall n \in\{1, \ldots, M-1\} \\
z^{0}=z_{0},
\end{array}\right.
$$

where $z_{0} \in \mathbb{R}^{\mathfrak{M}}, \mathcal{M}_{h}$ is the classical mass matrix and $\mathcal{A}_{h}$ is a suitable stiffness matrix approximating the fractional Laplacian. We are going to present more details on the construction of 4.6 in the next section.

In system [4.6), $v_{h, \delta t}=\left(v_{h}^{n}\right)_{1 \leq n \leq M}$ is a fully-discrete control function whose cost, that is the discrete $L_{\delta t}^{2}\left(0, T ; \mathbb{R}^{\mathfrak{M}}\right)$ norm, is defined by

$$
\left\|v_{\delta t}\right\|_{L_{\delta t}^{2}\left(0, T ; \mathbb{R}^{M}\right)}:=\left(\sum_{i=1}^{M} \delta t\left|v^{n}\right|_{L^{2}\left(\mathbb{R}^{\Re)}\right)}^{2}\right)^{1 / 2},
$$

and where $|\cdot|_{L^{2}\left(\mathbb{R}^{M)}\right)}$ stands for the norm associated to the $L^{2}$-inner product on $\mathbb{R}^{\mathfrak{M}}$

$$
(u, v)_{L^{2}\left(\mathbb{R}^{\Re}\right)}=h \sum_{i=1}^{N} u_{i} v_{i}
$$

With the above notation and according to the penalized HUM strategy, we introduce, for some penalization parameter $\varepsilon>0$, the following primal fully-discrete functional

$$
F_{\varepsilon, h, \delta t}\left(v_{\delta t}\right)=\frac{1}{2}\left\|v_{\delta t}\right\|_{L_{\delta t}^{2}\left(0, T ; \mathbb{R}^{\Re)}\right)}^{2}+\frac{1}{2 \varepsilon}\left|\mathcal{L}_{T}^{h}\left(v_{\delta t}, y_{0}\right)\right|_{L^{2}\left(\mathbb{R}^{\Re}\right)}^{2}, \quad \forall v_{\delta t} \in L_{\delta t}^{2}\left(0, T ; \mathbb{R}^{\mathfrak{M}}\right),
$$

that we wish to minimize onto the whole fully-discrete control space $L_{\delta t}^{2}\left(0, T ; \mathbb{R}^{\mathfrak{M}}\right)$ and where $z^{M}$ is the final value of the controlled problem [4.6. Here $\mathcal{L}_{T}^{h}(\cdot, \cdot)$ stands for the discrete version of the operator (4.2).

We can apply Fenchel-Rockafellar theory results to obtain the corresponding dual functional, which reads as follows

$$
J_{\varepsilon, h, \delta t}\left(\varphi^{T}\right)=\frac{1}{2}\left\|\mathcal{L}_{T}^{h}(\cdot, 0)^{\star} \varphi^{T}\right\|_{L_{\delta t}^{2}\left(0, T ; \mathbb{R}^{\Re)}\right)}^{2}+\frac{\varepsilon}{2}\left|\varphi^{T}\right|_{L^{2}\left(\mathbb{R}^{\Re}\right)}^{2}+\left(\varphi^{T}, \mathcal{L}_{T}^{h}\left(0, y_{0}\right)\right)_{L^{2}\left(\mathbb{R}^{\Re)}\right)}, \quad \forall \varphi^{T} \in L_{h, \delta t}^{2}(-1,1)
$$


where $\mathcal{L}_{T}^{h}(\cdot, \cdot)^{\star}$ is the adjoint of $\mathcal{L}_{T}^{h}(\cdot, \cdot)$ and

$$
\mathcal{L}_{T}^{h}(\cdot, 0)^{\star} \varphi^{T}=\left(\mathbf{1}_{\omega} \varphi^{n}\right)_{1 \leq n \leq M+1}
$$

with $\varphi=\left(\varphi^{n}\right)_{1 \leq n \leq M+1}$ solution to the adjoint system

$$
\left\{\begin{array}{l}
\mathcal{M}_{h} \frac{\varphi^{n}-\varphi^{n+1}}{\delta t}+\mathcal{A}_{h} \varphi^{n}=0, \quad \forall n \in\{1, \ldots, M\} \\
\varphi^{M+1}=\varphi^{T}
\end{array}\right.
$$

Notice that 4.7) is the fully-discrete approximation of 4.5). Moreover, it can be readily verified that this functional has a unique minimizer without any additional assumption on the problem. Therefore, by minimizing (4.7), and from duality theory, we obtain a control function

$$
v_{\varepsilon, h, \delta t}=\left(\mathbf{1}_{\omega} \varphi_{\varepsilon, h, \delta t}^{n}\right)_{1 \leq n \leq M},
$$

where $\varphi_{\epsilon}$ is the solution to (4.8) evaluated in the optimal datum $\varphi_{\varepsilon}^{T}$.

Thus, the optimal penalized control always exists and is unique. Deducing controllability properties amounts to study the behavior of this control with respect to the penalization parameter $\varepsilon$, in connection with the discretization parameters.

It is well known that, in general, we cannot expect for a given bounded family of initial data that the fully-discrete controls are uniformly bounded when the discretization parameters $h, \delta t$ and the penalization term $\varepsilon$ tend to zero independently. Instead, we expect to obtain uniform bounds by taking the penalization parameter $\varepsilon=\phi(h)$ that tends to zero in connection with the mesh size not too fast (see [12]) and a time step $\delta t$ verifying some weak condition of the kind $\delta t \leq \zeta(h)$ where $\zeta$ tends to zero logaritmically when $h \rightarrow 0$ (see [14]). This fact will be confirmed by the numerical simulations that we are going to present in Section 5.1 below, by observing the behavior of the norm of the control, the optimal energy inf $F_{\varepsilon}$, and the norm of the solution at time $T$. In this way, with the help of Theorem 4.1. we obtain numerical evidences of the properties of null and approximate controllability for equation (1.1), which are in accordance with the theoretical results in Section 2 .

\subsection{Finite element approximation}

According to the formulation of systems (4.6) and (4.8), in order to solve numerically our control problem, a proper approximation $\mathcal{A}_{h}$ of the operator $\left(-d_{x}^{2}\right)^{s}$ is needed. Furthermore, observe that, for every every time step $n$, (4.6) and 4.8) are actually discrete equations of the form

$$
\left(I+\delta t \mathcal{M}_{h}^{-1} \mathcal{A}_{h}\right) u_{h}=f_{h}
$$

The matrices appearing in the above equation will be computed employing a finite element scheme on a uniform mesh.

\section{Computation of the stiffness matrix $\mathcal{A}_{h}$.}

First of all, we recall the variational formulation associated to the elliptic equation $[1.3)$ : find $u \in H_{0}^{s}(-1,1)$ such that

$$
a(u, v)=\int_{-1}^{1} f v d x
$$

for all $v \in H_{0}^{s}(-1,1)$, where $a(u, v)$ is the bilinear form introduced in 2.4.

Let us take a partition of the interval $(-1,1)$ as follows:

$$
-1=x_{0}<x_{1}<\ldots<x_{i}<x_{i+1}<\ldots<x_{N+1}=1,
$$


with $x_{i+1}=x_{i}+h, i=0, \ldots N$. We call $\mathfrak{M}$ the mesh composed by the points $\left\{x_{i}: i=1, \ldots, N\right\}$, while the set of the boundary points is denoted $\partial \mathfrak{M}:=\left\{x_{0}, x_{N+1}\right\}$. Now, define $K_{i}:=\left[x_{i}, x_{i+1}\right]$ and consider the discrete space

$$
V_{h}:=\left\{v \in H_{0}^{s}(-1,1)|v|_{K_{i}} \in \mathcal{P}^{1}\right\}
$$

where $\mathcal{P}^{1}$ is the space of the continuous and piece-wise linear functions. Hence, we approximate (4.9) with the following discrete problem: find $u_{h} \in V_{h}$ such that

$$
\frac{c_{1, s}}{2} \int_{\mathbb{R}} \int_{\mathbb{R}} \frac{\left(u_{h}(x)-u_{h}(y)\right)\left(v_{h}(x)-v_{h}(y)\right)}{|x-y|^{1+2 s}} d x d y=\int_{-1}^{1} f v_{h} d x,
$$

for all $v_{h} \in V_{h}$. If now we indicate with $\left\{\phi_{i}\right\}_{i=1}^{N}$ a basis of $V_{h}$, it will be sufficient that the above equality is satisfied for all the functions of the basis, since any element of $V_{h}$ is a linear combination of them. Therefore the problem takes the following form

$$
\frac{c_{1, s}}{2} \int_{\mathbb{R}} \int_{\mathbb{R}} \frac{\left(u_{h}(x)-u_{h}(y)\right)\left(\phi_{i}(x)-\phi_{i}(y)\right)}{|x-y|^{1+2 s}} d x d y=\int_{-1}^{1} f v_{h} d x, \quad i=1, \ldots, N .
$$

Clearly, since $u_{h} \in V_{h}$, we have

$$
u_{h}(x)=\sum_{j=1}^{N} u_{j} \phi_{j}(x)
$$

where the coefficients $u_{j}$ are, a priori, unknown. In this way, 4.11) is reduced to solve the linear system $\mathcal{A}_{h} u=F$, where the stiffness matrix $\mathcal{A}_{h} \in \mathbb{R}^{N \times N}$ has components

$$
a_{i, j}=\frac{c_{1, s}}{2} \int_{\mathbb{R}} \int_{\mathbb{R}} \frac{\left(\phi_{i}(x)-\phi_{i}(y)\right)\left(\phi_{j}(x)-\phi_{j}(y)\right)}{|x-y|^{1+2 s}} d x d y,
$$

while the vector $F \in \mathbb{R}^{N}$ is given by $F=\left(F_{1}, \ldots, F_{N}\right)$ with

$$
F_{i}=\left\langle f, \phi_{i}\right\rangle=\int_{-1}^{1} f \phi_{i} d x, \quad i=1, \ldots, N .
$$

Moreover, the basis $\left\{\phi_{i}\right\}_{i=1}^{N}$ that we will employ is the classical one in which each $\phi_{i}$ is the tent function with $\operatorname{supp}\left(\phi_{i}\right)=\left(x_{i-1}, x_{i+1}\right)$ and verifying $\phi_{i}\left(x_{j}\right)=\delta_{i, j}$. In particular, for $x \in\left\{x_{i-1}, x_{i}, x_{i+1}\right\}$ the $i^{\text {th }}$ function of the basis is explicitly defined as (see Figure 1 )

$$
\phi_{i}(x)=1-\frac{\left|x-x_{i}\right|}{h}
$$

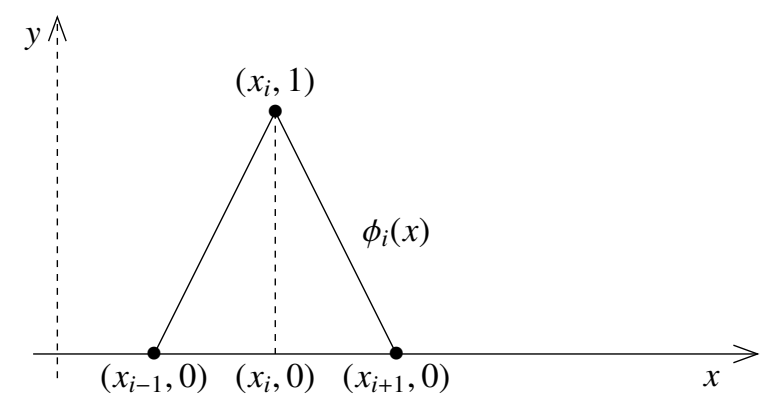

Figure 1: Basis function $\phi_{i}(x)$ on its support $\left(x_{i-1}, x_{i+1}\right)$.

Let us now describe our algorithm for computing the coefficients $a_{i, j}$. Before that, we shall make the following preliminary comments. 
Remark 4.1. The following fact are worth noticing.

1. The constant $c_{1, s}$ in the definition (2.1) of the fractional Laplacian is common for all the entries of the matrix. For the sake of simplicity, we will drop this constant in the following computations.

2. It is evident from the definition (4.12) that $\mathcal{A}_{h}$ is symmetric. Therefore, in our algorithm we will only need to compute the values $a_{i, j}$ with $j \geq i$.

3. Due to the non-local nature of the problem, the matrix $\mathcal{A}_{h}$ is full. However, while computing its components, we will encounter many simplifications, due to the fact that $\operatorname{supp}\left(\phi_{i}\right) \cap \operatorname{supp}\left(\phi_{j}\right)=\emptyset$ for $j \geq i+2$.

4. While computing the values $a_{i, j}$, we will only work on the mesh $\mathfrak{M}$, not considering the points of the set $\partial \mathfrak{M}$. In this way, we will ensure that the basis functions $\phi_{i}$ satisfy the zero Dirichlet boundary conditions. In other words, in our FE approximation we are considering only the functions from $\phi_{1}$ to $\phi_{N}$. Instead, if we considered the points $x_{0}$ and $x_{N+1}$, then we would need to introduce in our discretization also the basis functions $\phi_{0}$ and $\phi_{N+1}$, which take value one at the boundary, and this would not be consistent with the continuous problem. Figure 2 provides a graphical explanation of this last discussion.

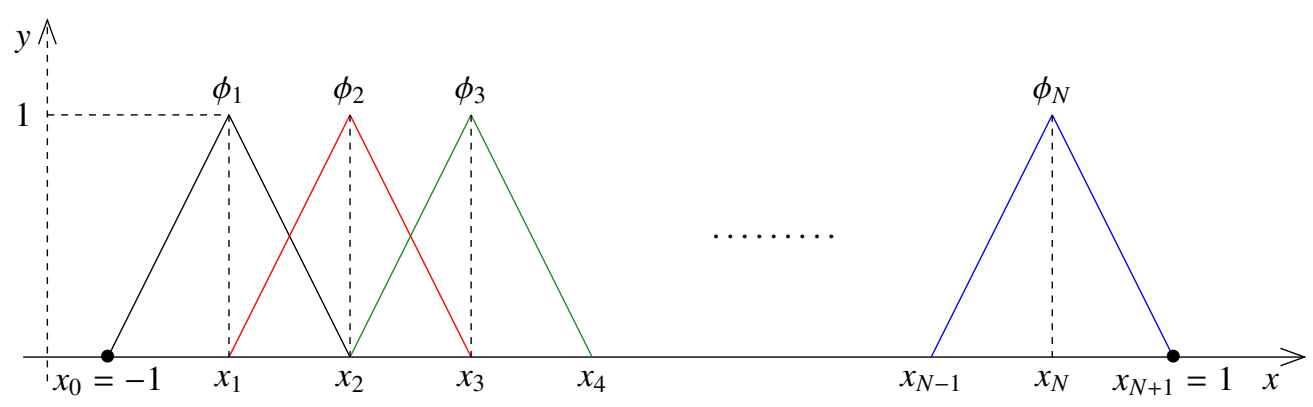

Figure 2: Basis functions $\phi_{i}(x)$ on the whole interval $(-L, L)$.

We now start building the stiffness matrix $\mathcal{A}_{h}$. This will be done it in three steps, since the values of the matrix can be computed differentiating among three well defined regions: the upper triangle, corresponding to $j \geq i+2$, the upper diagonal corresponding to $j=i+1$ and the diagonal, corresponding to $j=i$ (see Figure 3). In fact, as it will be clear during our computations, in each of these regions the intersections among the support of the basis functions are different, thus generating different values of the bilinear form. In what follows, we will briefly present which will be the contributions to the matrix in each of these three steps, including the complete computations as an appendix at the end of the paper.

Step 1: $j \geq i+2$

As we mentioned in Remark 4.1(3), in this case we have $\operatorname{supp}\left(\phi_{i}\right) \cap \operatorname{supp}\left(\phi_{j}\right)=\emptyset$ (see also Figure 4). Hence, (4.12) is reduced to computing only the integral

$$
a_{i, j}=-2 \int_{x_{j-1}}^{x_{j+1}} \int_{x_{i-1}}^{x_{i+1}} \frac{\phi_{i}(x) \phi_{j}(y)}{|x-y|^{1+2 s}} d x d y .
$$

Taking into account the definition of the basis function 4.13, from 4.14) we obtain

$$
a_{i, j}=-2 \int_{x_{j-1}}^{x_{j+1}} \int_{x_{i-1}}^{x_{i+1}} \frac{\left(1-\frac{\left|x-x_{i}\right|}{h}\right)\left(1-\frac{\left|y-x_{j}\right|}{h}\right)}{|x-y|^{1+2 s}} d x d y .
$$

Finally, this last integral can be computed explicitly employing the following change of variables:

$$
\frac{x-x_{i}}{h}=\hat{x}, \quad \frac{y-x_{i}}{h}=\hat{y} .
$$




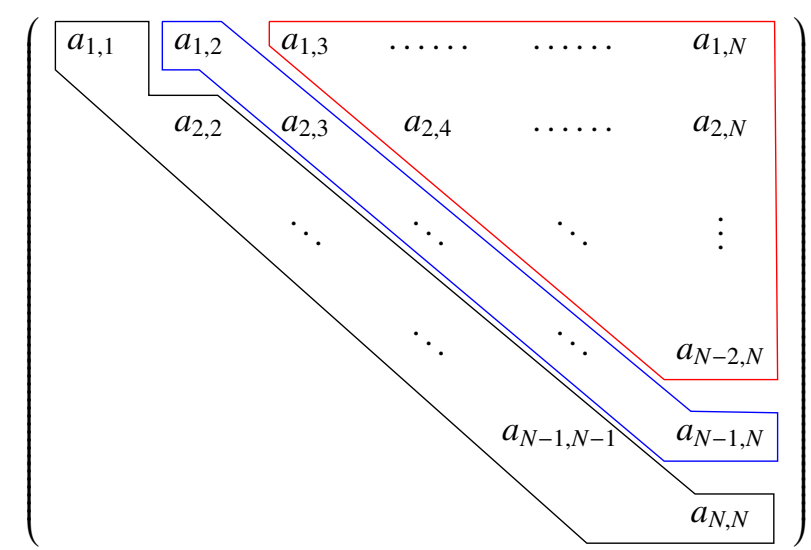

Figure 3: Structure of the stiffness matrix $\mathcal{A}_{h}$.

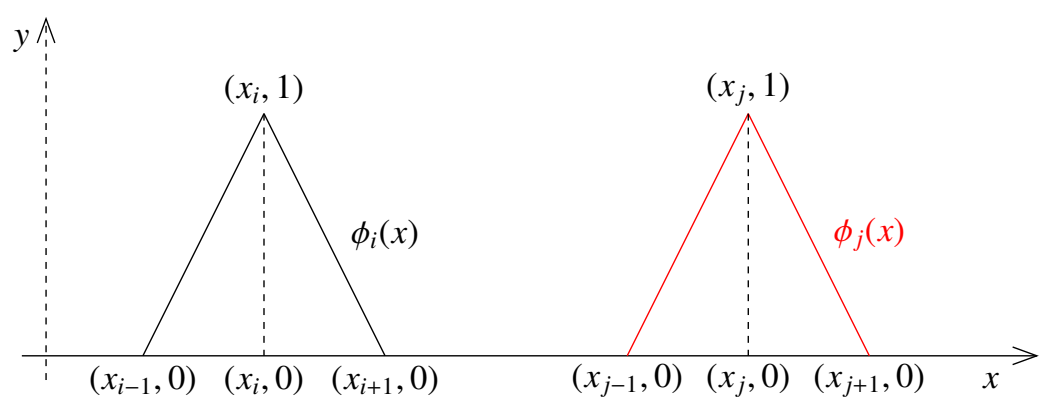

Figure 4: Basis functions $\phi_{i}(x)$ and $\phi_{j}(x)$ for $j \geq i+1$. In this case, the supports are disjoint.

In this way, for the elements $a_{i, j}, j \geq i+2$, we get the following values:

$$
a_{i, j}= \begin{cases}-h^{1-2 s} \frac{4(k+1)^{3-2 s}+4(k-1)^{3-2 s}-6 k^{3-2 s}-(k+2)^{3-2 s}-(k-2)^{3-2 s}}{2 s(1-2 s)(1-s)(3-2 s)}, & k=j-i, \quad s \neq \frac{1}{2} \\ -4(j-i+1)^{2} \log (j-i+1)-4(j-i-1)^{2} \log (j-i-1) & s=\frac{1}{2}, \quad j>i+2 \\ +6(j-i)^{2} \log (j-i)+(j-i+2)^{2} \log (j-i+2)+(j-i-2)^{2} \log (j-i-2), & \\ 56 \ln (2)-36 \ln (3), & s=\frac{1}{2}, \quad j=i+2 .\end{cases}
$$

Step 2: $j=i+1$

This is the most cumbersome case, since it is the one with the most interactions between the basis functions (see Figure 5). According to (4.12), and using the symmetry of the integral with respect to the bisector $y=x$, we have

$$
\begin{aligned}
a_{i, i+1}= & \int_{\mathbb{R}} \int_{\mathbb{R}} \frac{\left(\phi_{i}(x)-\phi_{i}(y)\right)\left(\phi_{i+1}(x)-\phi_{i+1}(y)\right)}{|x-y|^{1+2 s}} d x d y \\
= & \int_{x_{i+1}}^{+\infty} \int_{x_{i+1}}^{+\infty} \ldots d x d y+2 \int_{x_{i+1}}^{+\infty} \int_{x_{i}}^{x_{i+1}} \ldots d x d y+2 \int_{x_{i+1}}^{+\infty} \int_{-\infty}^{x_{i}} \ldots d x d y \\
& +\int_{x_{i}}^{x_{i+1}} \int_{x_{i}}^{x_{i+1}} \ldots d x d y+2 \int_{x_{i}}^{x_{i+1}} \int_{-\infty}^{x_{i}} \ldots d x d y+\int_{-\infty}^{x_{i}} \int_{-\infty}^{x_{i}} \ldots d x d y
\end{aligned}
$$




$$
:=Q_{1}+Q_{2}+Q_{3}+Q_{4}+Q_{5}+Q_{6}
$$

These contributions will be calculated separately, employing changes of variables analogous to (4.15). After several

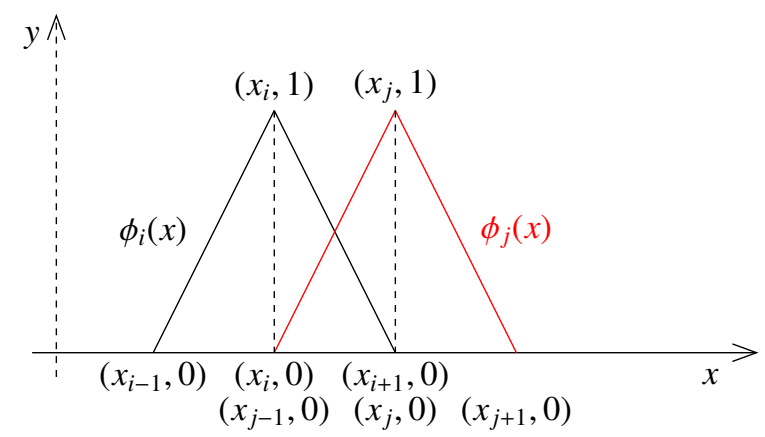

Figure 5: Basis functions $\phi_{i}(x)$ and $\phi_{i+1}(x)$. In this case, the intersection of the supports is the interval $\left[x_{i}, x_{i+1}\right]$. computations, we obtain

$$
a_{i, i+1}= \begin{cases}h^{1-2 s} \frac{3^{3-2 s}-2^{5-2 s}+7}{2 s(1-2 s)(1-s)(3-2 s)}, & s \neq \frac{1}{2} \\ 9 \ln 3-16 \ln 2, & s=\frac{1}{2} .\end{cases}
$$

Step 3: $j=i$

As a last step, we fill the diagonal of the matrix $\mathcal{A}_{h}$, which collects the values corresponding to the case $\phi_{i}(x)=\phi_{j}(x)$ (see Figure 6). We have

$$
\begin{aligned}
& a_{i, i}=\int_{\mathbb{R}} \int_{\mathbb{R}} \frac{\left(\phi_{i}(x)-\phi_{i}(y)\right)^{2}}{|x-y|^{1+2 s}} d x d y \\
& =\int_{x_{i+1}}^{+\infty} \int_{x_{i+1}}^{+\infty} \ldots d x d y+2 \int_{x_{i+1}}^{+\infty} \int_{x_{i-1}}^{x_{i+1}} \ldots d x d y+\int_{x_{i+1}}^{+\infty} \int_{-\infty}^{x_{i-1}} \ldots d x d y \\
& +\int_{x_{i-1}}^{x_{i+1}} \int_{x_{i-1}}^{x_{i+1}} \ldots d x d y+2 \int_{-\infty}^{x_{i-1}} \int_{x_{i-1}}^{x_{i+1}} \ldots d x d y++\int_{-\infty}^{x_{i-1}} \int_{x i+1}^{+\infty} \ldots d x d y \\
& +\int_{-\infty}^{x_{i-1}} \int_{-\infty}^{x_{i-1}} \ldots d x d y:=R_{1}+R_{2}+R_{3}+R_{4}+R_{5}+R_{6}+R_{7} .
\end{aligned}
$$

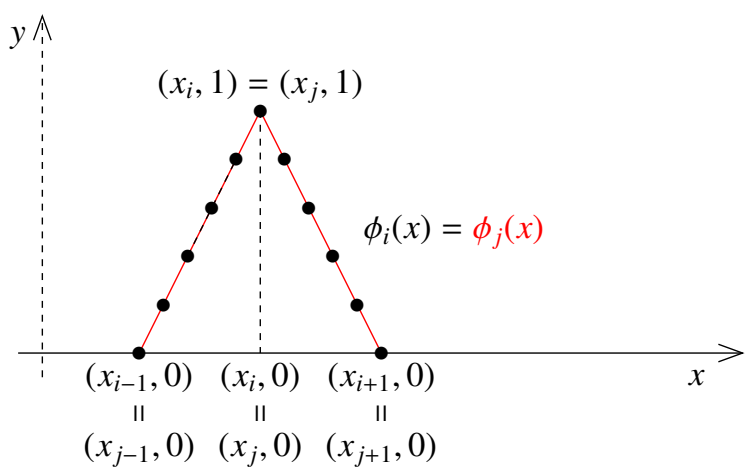

Figure 6: Basis functions $\phi_{i}(x)$ and $\phi_{j}(x)$. In this case, the two functions coincide. 
Once again, the terms $R_{i}, i=1, \ldots, 7$ will be computed separately, and summing them we will obtain

$$
a_{i, i}= \begin{cases}h^{1-2 s} \frac{2^{3-2 s}-4}{s(1-2 s)(1-s)(3-2 s)}, & s \neq \frac{1}{2} \\ 8 \ln 2, & s=\frac{1}{2} .\end{cases}
$$

\section{Conclusion}

Summarizing, we have the following values for the elements of the stiffness matrix $\mathcal{A}_{h}$ : for $s \neq 1 / 2$

$$
a_{i, j}=-h^{1-2 s} \begin{cases}\frac{4(k+1)^{3-2 s}+4(k-1)^{3-2 s}-6 k^{3-2 s}-(k+2)^{3-2 s}-(k-2)^{3-2 s}}{2 s(1-2 s)(1-s)(3-2 s)}, & k=j-i, k \geq 2 \\ \frac{3^{3-2 s}-2^{5-2 s}+7}{2 s(1-2 s)(1-s)(3-2 s)}, & j=i+1 \\ \frac{2^{3-2 s}-4}{s(1-2 s)(1-s)(3-2 s)}, & j=i .\end{cases}
$$

For $s=1 / 2$, instead, we have

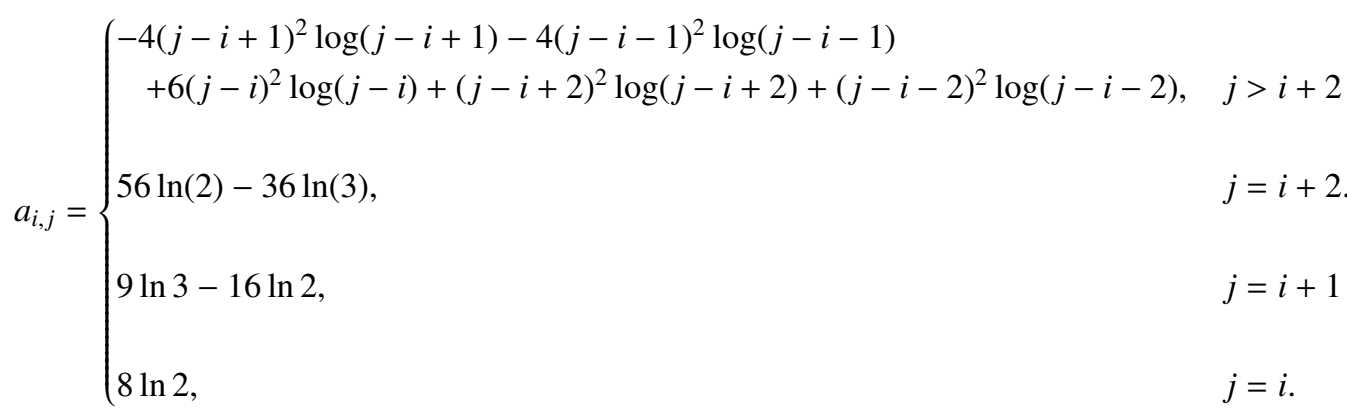

Remark 4.2. We point out the following facts:

1. The matrix $\mathcal{A}_{h}$ has the structure of a $N$-diagonal matrix, meaning that value of its elements remain constant along its diagonals. This is in analogy with the tridiagonal matrix approximating the classical Laplace operator. Notice, however, that in our case we obtain a full matrix. This is consistent with the nonlocal nature of the operator that we are discretizing.

2. The value of each element $a_{i, j}$ is given explicitly, and it only depends on $i, j, s$ and $h$. In other words, when approximating the left hand side of 4.11, no numerical integration is needed.

3. For $s=1 / 2$, the elements $a_{i, j}$ do not depend on the value of $h$ which, in turn, is a function of $N$. This implies that, in this particular case, no matter how many points we consider in our mesh, the matrix $\mathcal{A}_{h}$ will always have the same entries.

4. A quick computation shows that taking the limit $s \rightarrow 1^{-}$in $\mathcal{A}_{h}$ we recover the tridiagonal matrix of the classical FE approximation of the local Poisson equation. This is in accordance with the same behavior of the continuous operator, as shown, for instance, in [18] Proposition 4.4]. 


\section{Computation of the mass matrix $\mathcal{M}_{h}$.}

The matrix $\mathcal{M}_{h}$ appears when deriving the FE scheme for the parabolic problem (1.1). Adapting the same construction that we presented before for the elliptic equation (1.3), the approximate solution $z_{h}$ shall be written in the form

$$
z_{h}(x, t):=\sum_{j=1}^{N} z_{j}(t) \phi_{j}(x),
$$

where the basis functions $\left\{\phi_{j}\right\}_{j=1}^{N}$ are the same as before while the coefficients $\left\{z_{j}(t)\right\}_{j=1}^{N}$ have to be computed. Then, the corresponding variational formulation reads as

$$
\sum_{j=1}^{N}(z_{j}^{\prime}(t) \underbrace{\int_{-1}^{1} \phi_{j} \phi_{i} d x}_{m_{i, j}}+z_{j}(t) \underbrace{a\left(\phi_{j}, \phi_{i}\right)}_{a_{i, j}})=\underbrace{\int_{\omega} g \phi_{i} d x}_{g_{i}(t)}, \quad i=1, \ldots, N .
$$

Therefore, denoting $g(t):=\left(g_{1}(t), \ldots, g_{N}(t)\right)^{T}$, the unknown vector $z(t):=\left(z_{1}(t), \ldots, z_{N}(t)\right)^{T}$ is obtained by solving the following linear system

$$
\mathcal{M}_{h} z^{\prime}(t)+\mathcal{A}_{h} z(t)=g(t)
$$

where $\mathcal{M}_{h}$ is the mass matrix with entries $m_{i, j}$ and $\mathcal{A}_{h}$ is the stiffness matrix composed by the entries $a_{i, j}$ previously computed.

Solving (4.16) by means of an implicit Euler method in time, we obtain exactly the formulation (4.6). Moreover, (4.8) is obtained with the same procedure, taking into account that this time, the original problem being homogeneous, the vector $g$ is actually zero.

\section{Numerical results}

In this Section, we present the numerical simulations corresponding to the algorithm previously described, and we provide a complete discussion of the results obtained.

First of all, we test numerically the accuracy of our method for the resolution of the elliptic equation $[1.3$, by applying it to the following problem

$$
\begin{cases}\left(-d_{x}^{2}\right)^{s} u=1, & x \in(-1,1) \\ u \equiv 0, & x \in \mathbb{R} \backslash(-1,1) .\end{cases}
$$

In this particular case, the unique solution to [5.1) can be computed exactly and it is given in [26]. It reads as follows,

$$
u(x)=\frac{2^{-2 s} \sqrt{\pi}}{\Gamma\left(\frac{1+2 s}{2}\right) \Gamma(1+s)}\left(1-x^{2}\right)^{s} \cdot \mathbf{1}_{(-1,1)} .
$$

In Figure 7, we show a comparison for different values of $s$ between the exact solution 5.2 and the computed numerical approximation. Here we consider $N=50$. One can notice that when $s=0.1$ (and also for other small values of s), the computed solution is to a certain extent different from the exact solution. However, one should be careful with such result and a more precise analysis of the error should be carried.

In the same spirit as in [2], the computation of the error in the space $H_{0}^{s}(-1,1)$ can be readily done by using the definition of the bilinear form, namely

$$
\left\|u-u_{h}\right\|_{H_{0}^{s}(-1,1)}^{2}=a\left(u-u_{h}, u-u_{h}\right)=a\left(u, u-u_{h}\right)=\int_{-1}^{1} f(x)\left(u(x)-u_{h}(x)\right) d x,
$$




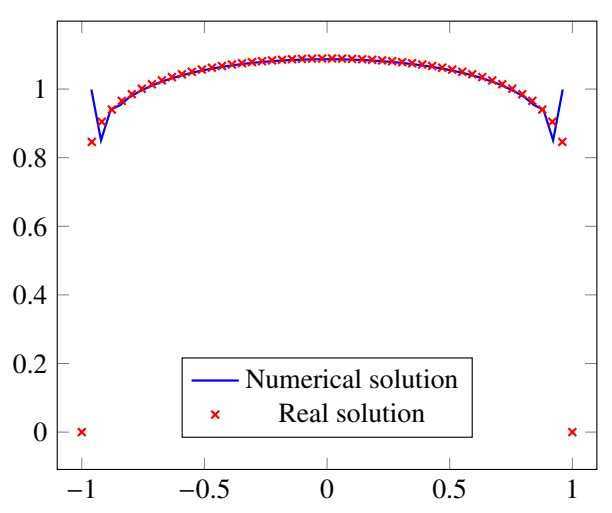

(a) $s=0.1$

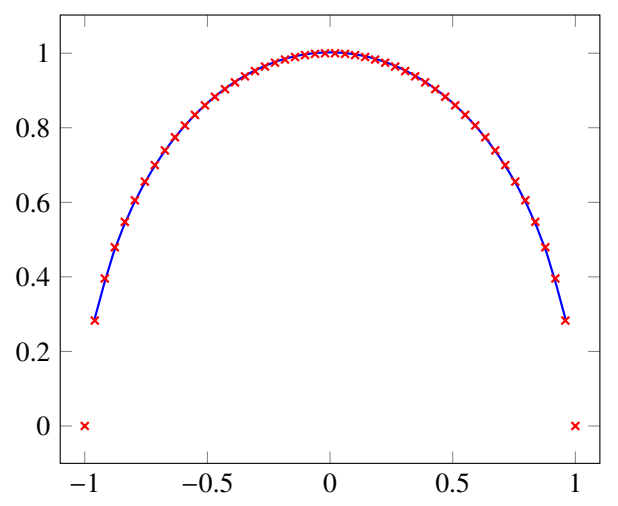

(c) $s=0.5$

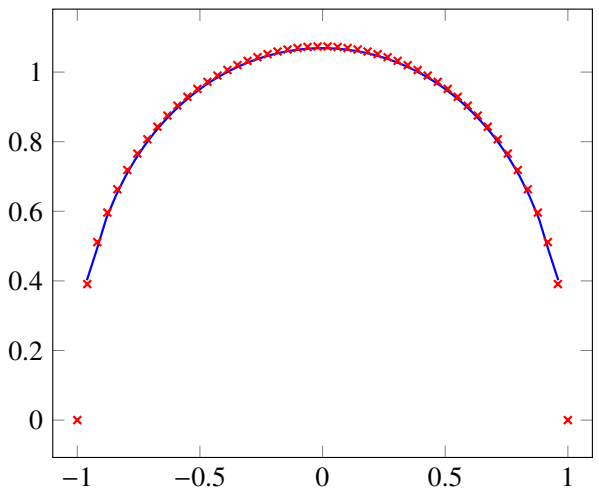

(b) $s=0.4$

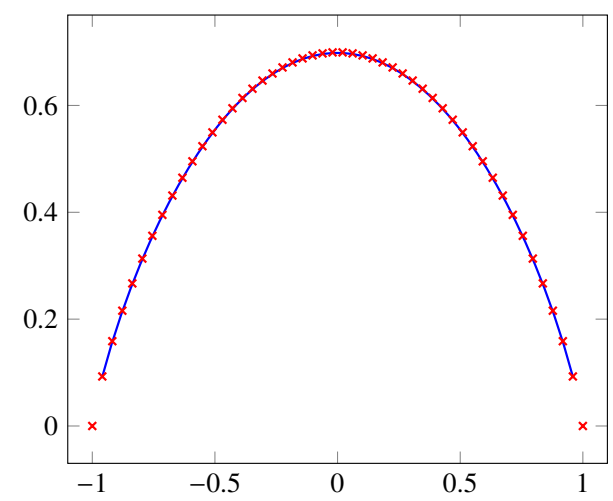

(d) $s=0.8$

Figure 7: Plot for different values of $s$.

where have used the orthogonality condition $a\left(v_{h}, u-u_{h}\right)=0 \forall v_{h} \in V_{h}$.

For this particular test, since $f \equiv 1$ in $(-1,1)$, the problem is therefore reduced to

$$
\left\|u-u_{h}\right\|_{H_{0}^{s}(-1,1)}=\left(\int_{-1}^{1}\left(u(x)-u_{h}(x)\right) d x\right)^{1 / 2}
$$

where the right-hand side can be easily computed, since we have the closed formula

$$
\int_{-1}^{1} u d x=\frac{\pi}{2^{2 s} \Gamma\left(s+\frac{1}{2}\right) \Gamma\left(s+\frac{3}{2}\right)}
$$

and the term corresponding to $\int_{-1}^{1} u_{h}$ can be carried out numerically.

In Figure 8 , we present the computational errors evaluated for different values of $s$ and $h$.

The rates of convergence shown are of order (in $h$ ) of $1 / 2$. This is in accordance with the following result:

Theorem 5.1 ([2, Theorem 4.6]). For the solution $u$ of (4.9) and its FE approximation $u_{h}$ given by [4.11], if $h$ is sufficiently small, the following estimates hold

$$
\begin{array}{llrl}
\left\|u-u_{h}\right\|_{H_{0}^{s}(-1,1)} \leq C h^{1 / 2}|\ln h|\|f\|_{C^{1 / 2-s}(-1,1)}, & \text { if } \quad s<1 / 2, \\
\left\|u-u_{h}\right\|_{H_{0}^{s}(-1,1)} \leq C h^{1 / 2}|\ln h|\|f\|_{L^{\infty}(-1,1)}, & \text { if } \quad s=1 / 2 \\
\left\|u-u_{h}\right\|_{H_{0}^{s}(-1,1)} \leq \frac{C}{2 s-1} h^{1 / 2} \sqrt{|\ln h|}\|f\|_{C^{\beta}(-1,1)}, & \text { if } \quad s>1 / 2, \quad \beta>0
\end{array}
$$




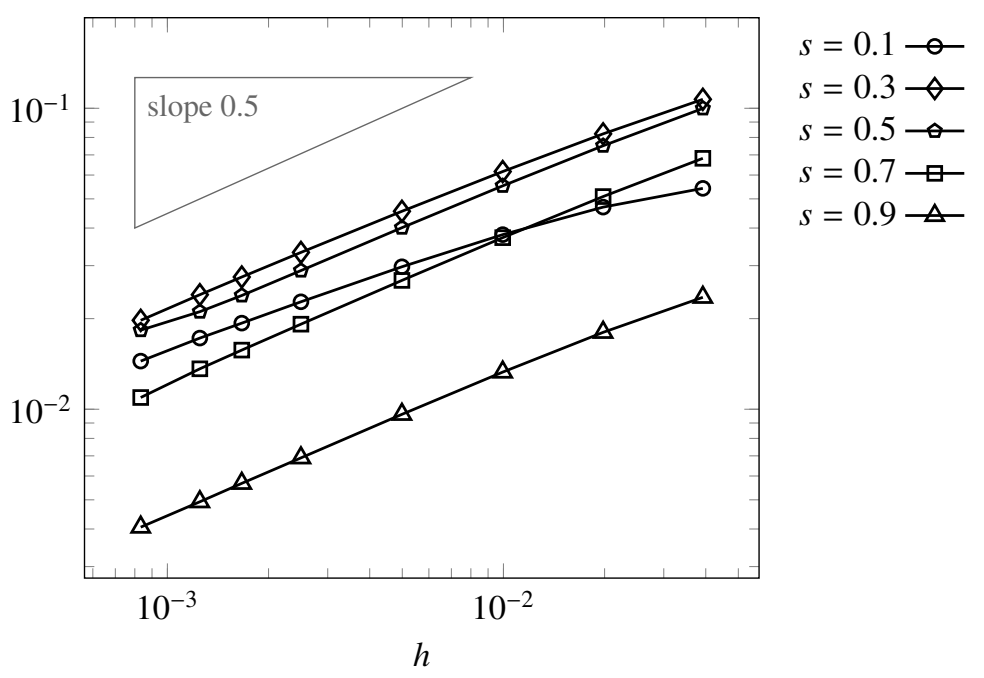

Figure 8: Convergence of the error.

where $C$ is a positive constant not depending on $h$.

Moreover, Figure 8 shows that the convergence rate is maintained also for small values of $s$. This confirms that the behavior shown in Figure $7 \mathrm{a}$ is not in contrast with the known theoretical results. Indeed, since it is well-known that the notion of trace is not defined for the spaces $H^{s}(-1,1)$ with $s \leq 1 / 2$ (see [36, 47]), it is somehow natural that we cannot expect a point-wise convergence in this case.

\subsection{Control experiments}

To address the actual computation of fully-discrete controls for a given problem, we use the methodology described, for instance, in [29]. We apply an optimization algorithm to the dual functional 4.7]. Since these functionals are quadratic and coercive, the conjugate gradient is a natural and quite simple choice.

First of all, starting from the expression (4.7p, the gradient of the functional $J_{\varepsilon, h, \delta t}\left(\varphi^{T}\right)$ can be easily computed and it reads as

$$
\nabla J_{\varepsilon, h, \delta t}\left(\varphi^{T}\right)=\mathcal{L}_{T}^{h}\left(\mathcal{L}_{T}^{h}(\cdot, 0)^{\star} \varphi^{T}, 0\right)+\varepsilon \varphi^{T}+\mathcal{L}_{T}^{h}\left(0, y_{0}\right)
$$

Hence, the computation of this gradient at each iteration amounts to solve first the homogeneous equation (4.8). Then, set $v^{n}=\varphi^{n} \mathbf{1}_{\omega}$ and finally solve (4.6) with zero initial datum. In this way, the procedure to compute the gradient of (4.7) basically requires to solve two parabolic equations: a homogeneous backward one associated with the final data $\varphi^{T}$, and a non-homogeneous forward problem with zero initial data.

We present now some results obtained with the described methodology. In accordance with the discussion in Section 4, we use the finite-element approximation of $\left(-d_{x}^{2}\right)^{s}$ for the space discretization and the implicit Euler scheme in the time variable. We denote by $N$ the number of points in the mesh and by $M$ the number of time intervals. As discussed in [14], the results in this kind of problems does not depend too much in the time step, as soon as it is chosen to ensure at least the same accuracy as the space discretization. The same remains true here, and therefore we always take $M=2000$ in order to concentrate the discussion on the dependency of the results with respect to the mesh size $h$ and the parameter $s$.

As we mentioned, we choose the penalization term $\varepsilon$ as a function of $h$. A reasonable practical rule ([12]) is to systematically choose $\varepsilon=\phi(h) \sim h^{2 p}$ where $p$ is the order of accuracy in space of the numerical method employed for the discretization of the spatial operator involved (in this case the fractional Laplacian (2.1)).

We recall that the solution $z$ to 1.1 belongs to the space $L^{2}\left(0, T ; H_{0}^{s}(-1,1)\right) \cap C\left([0, T] ; L^{2}(-1,1)\right)$. In view of that, we immediately have that $z(\cdot, T) \in L^{2}(-1,1)$. Therefore, we shall choose the value of $p$ as the convergence rate in the $L^{2}$-norm for the discretization of the elliptic problem (1.3). This convergence rate is given by the following result. 
Theorem 5.2 ([11, Proposition 3.3.2]). Let $s \in(0,1), f \in L^{2}(-1,1)$ and $u$ be the solution to (1.3). Given a uniform mesh $\mathfrak{M}$ with mesh size $h$, and the space $V_{h}$ defined as in 4.10 , let $u_{h}$ be the finite element solution to the corresponding discrete problem. Then, it holds that

$$
\left\|u-u_{h}\right\|_{L^{2}(-1,1)} \leq C(s, \alpha) h^{2 \alpha}\|f\|_{L^{2}(-1,1)},
$$

where $\alpha:=\min \{s, 1 / 2-\varepsilon\}$, for all $\varepsilon>0$.

By virtue of Theorem 5.2 the appropriate value of $p$ that we shall employ is

$$
p=2 \alpha= \begin{cases}2 s, & \text { for } s<\frac{1}{2} \\ 1-2 \varepsilon, & \text { for } s \geq \frac{1}{2} .\end{cases}
$$

We present below the numerical experiments obtained applying our method. We begin by plotting on Figure 9 the time evolution of the uncontrolled solution as well as the controlled solution. Here, we set $s=0.8, \omega=(-0.3,0.8)$ and $T=0.3$, and as an initial condition we take $z_{0}(x)=\sin (\pi x)$. The control domain is represented as highlighted zone on the plane $(t, x)$. As expected, we observe that the uncontrolled solution is damped with time, but does not reach zero at time $T$, while the controlled solution does.

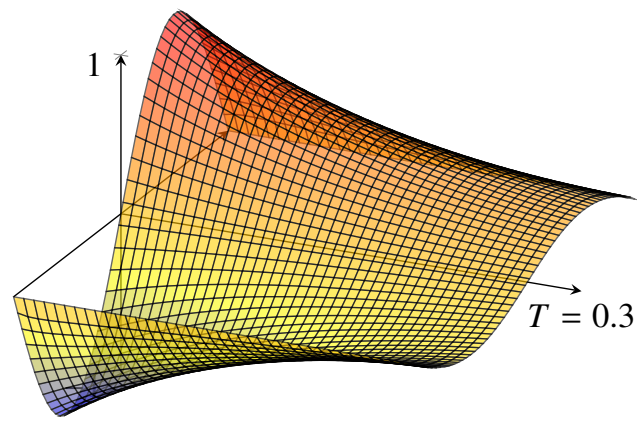

(a) Uncontrolled solution

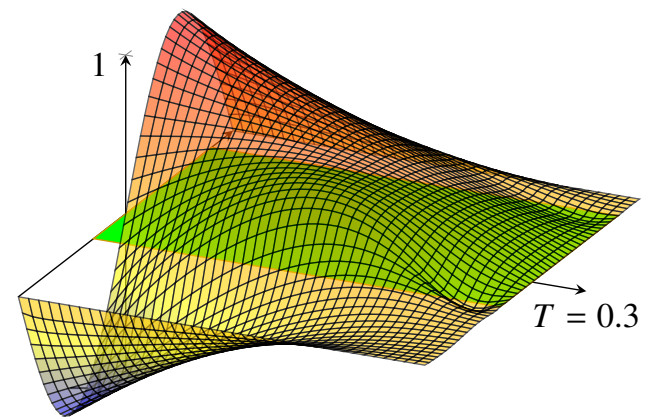

(b) Controlled solution ( $\square=$ control domain)

Figure 9: Time evolution of system 4.6.

In Figure 10, we present the computed values of various quantities of interest when the mesh size goes to zero. More precisely, we observe that the control cost $\left\|v_{\delta t}\right\|_{L_{\delta t}^{2}\left(0, T ; \mathbb{R}^{\Re)}\right)}$ and the optimal energy remain bounded as $h \rightarrow 0$. On the other hand, we see that

$$
\left|y^{M}\right|_{L^{2}\left(\mathbb{R}^{M}\right)} \sim C \sqrt{\phi(h)}=C h^{1 / 2} .
$$

We know that, for $s=0.8$, system (1.1) is null controllable. This is now confirmed by [5.3), according to Theorem 4.1. In fact, the same experiment can be repeated for different values of $s>1 / 2$, obtaining the same conclusions.

According to the discussion in Section 2, one can prove that null controllability does not hold for system (1.1) in the case $s \leq 1 / 2$. However approximate controllability can be proved by means of the unique continuation property of the operator $\left(-d_{x}^{2}\right)^{s}$. We would like to illustrate this property in Figure 11 .

We observe that the results are different from what we obtained in Figure 10 In fact, the cost of the control and the optimal energy increase in both cases, while the target $y^{M}$ tends to zero with a slower rate than $h^{1 / 2}$. This seems to confirm that a uniform observability estimate for (1.1) does not hold and that we can only expect to have approximate controllability (see Theorem 4.1).

\section{A Explicit computations of the elements of the matrix $\mathcal{A}_{h}$}

We present here the explicit computations for each element $a_{i, j}$ of the stiffness matrix, completing the discussion that we started in Section 4 


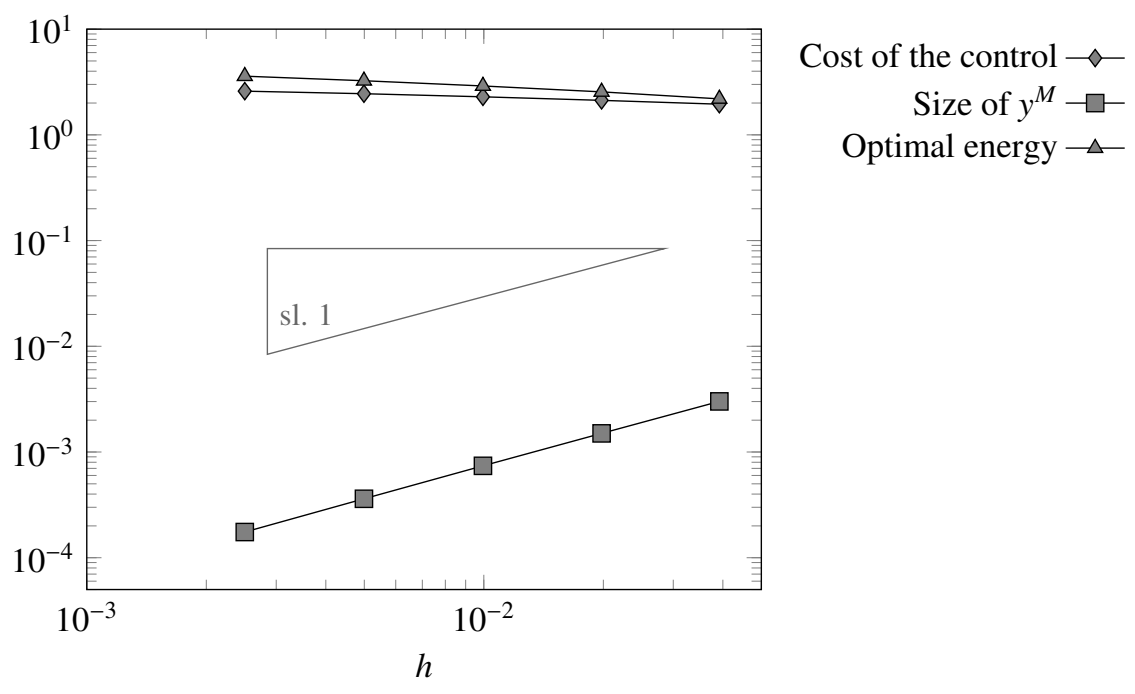

Figure 10: Convergence properties of the method for the controllability of the fractional heat equation for $s=0.8$.

Step 1: $j \geq i+2$

We recall that, in this case, the value of $a_{i, j}$ is given by the integral

$$
a_{i, j}=-2 \int_{x_{j-1}}^{x_{j+1}} \int_{x_{i-1}}^{x_{i+1}} \frac{\phi_{i}(x) \phi_{j}(y)}{|x-y|^{1+2 s}} d x d y .
$$

In Figure 12, we give a scheme of the region of interaction (marked in gray) between the basis functions in this case. These are the only regions in which (A.1) will be different than zero.

Now, taking into account the definition of the basis function 4.13, the integral A.1) becomes

$$
a_{i, j}=-2 \int_{x_{j-1}}^{x_{j+1}} \int_{x_{i-1}}^{x_{i+1}} \frac{\left(1-\frac{\left|x-x_{i}\right|}{h}\right)\left(1-\frac{\left|y-x_{j}\right|}{h}\right)}{|x-y|^{1+2 s}} d x d y .
$$

Let us introduce the following change of variables:

$$
\frac{x-x_{i}}{h}=\hat{x}, \quad \frac{y-x_{i}}{h}=\hat{y} .
$$

Then, rewriting (with some abuse of notations since there is no possibility of confusion) $\hat{x}=x$ and $\hat{y}=y$, we get

$$
a_{i, j}=-2 h^{1-2 s} \int_{-1}^{1} \int_{-1}^{1} \frac{(1-|x|)(1-|y|)}{|x-y+i-j|^{1+2 s}} d x d y .
$$

The integral A.2 can be computed explicitly in the following way. First of all, for simplifying the notation, let us define $k=j-i$. We have

$$
\begin{aligned}
a_{i, j}= & -2 h^{1-2 s} \int_{-1}^{1} \int_{-1}^{1} \frac{(1-|x|)(1-|y|)}{|x-y+i-j|^{1+2 s}} d x d y=-2 h^{1-2 s} \int_{-1}^{1} \int_{-1}^{1} \frac{(1-|x|)(1-|y|)}{|x-y-k|^{1+2 s}} d x d y \\
= & -2 h^{1-2 s} \int_{0}^{1} \int_{0}^{1} \frac{(1-x)(1-y)}{(y-x+k)^{1+2 s}} d x d y-2 h^{1-2 s} \int_{0}^{1} \int_{-1}^{0} \frac{(1+x)(1-y)}{(y-x+k)^{1+2 s}} d x d y \\
& -2 h^{1-2 s} \int_{-1}^{0} \int_{0}^{1} \frac{(1-x)(1+y)}{(y-x+k)^{1+2 s}} d x d y-2 h^{1-2 s} \int_{-1}^{0} \int_{-1}^{0} \frac{(1+x)(1+y)}{(y-x+k)^{1+2 s}} d x d y
\end{aligned}
$$




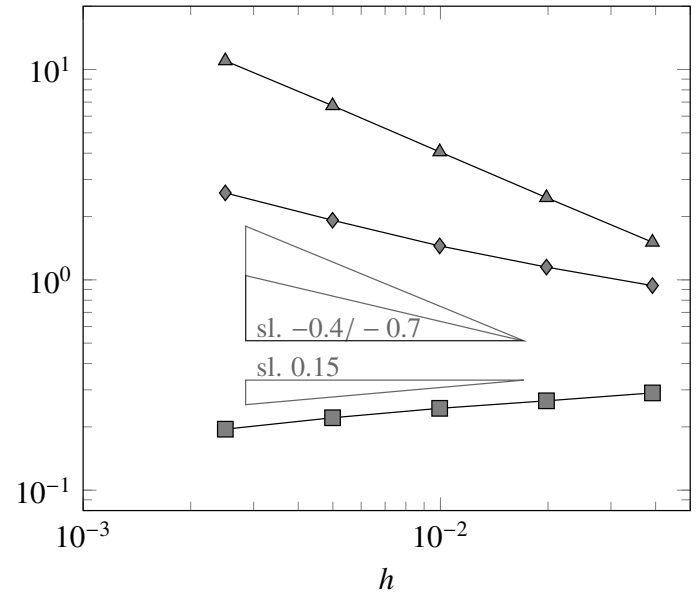

(a) $s=0.4$

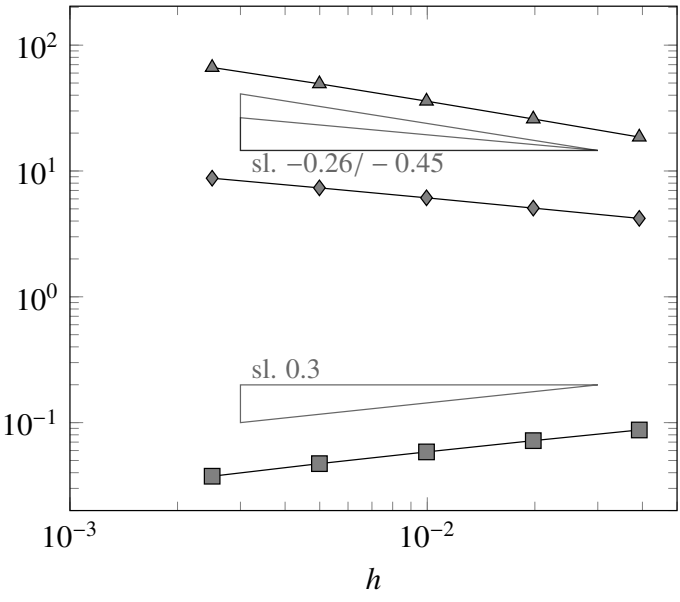

(b) $s=0.5$

Figure 11: Convergence properties of the method for $s<1 / 2$. Same legend as in Figure 10

$$
=-2 h^{1-2 s}\left(B_{1}+B_{2}+B_{3}+B_{4}\right) .
$$

These terms $B_{i}, i=1,2,3,4$, can be computed integrating by parts several times. In more detail, we have

$$
\begin{aligned}
& B_{1}=\frac{1}{4 s(1-2 s)}\left[2 k^{1-2 s}-\frac{(k+1)^{2-2 s}-(k-1)^{2-2 s}}{1-s}-\frac{2 k^{3-2 s}-(k+1)^{3-2 s}-(k-1)^{3-2 s}}{(1-s)(3-2 s)}\right] \\
& B_{2}=\frac{1}{4 s(1-2 s)}\left[-2 k^{1-2 s}+\frac{2(k+1)^{2-2 s}-2 k^{2-2 s}}{1-s}+\frac{2(k+1)^{3-2 s}-k^{3-2 s}-(k+2)^{3-2 s}}{(1-s)(3-2 s)}\right] \\
& B_{3}=\frac{1}{4 s(1-2 s)}\left[-2 k^{1-2 s}+\frac{2 k^{2-2 s}-2(k-1)^{2-2 s}}{1-s}+\frac{2(k-1)^{3-2 s}-k^{3-2 s}-(k-2)^{3-2 s}}{(1-s)(3-2 s)}\right] \\
& B_{4}=\frac{1}{4 s(1-2 s)}\left[2 k^{1-2 s}-\frac{(k+1)^{2-2 s}-(k-1)^{2-2 s}}{1-s}-\frac{2 k^{3-2 s}-(k+1)^{3-2 s}-(k-1)^{3-2 s}}{(1-s)(3-2 s)}\right] .
\end{aligned}
$$

Therefore, we obtain

$$
a_{i, j}=-h^{1-2 s} \frac{4(k+1)^{3-2 s}+4(k-1)^{3-2 s}-6 k^{3-2 s}-(k+2)^{3-2 s}-(k-2)^{3-2 s}}{2 s(1-2 s)(1-s)(3-2 s)} .
$$

We notice that, when $s=1 / 2$, both the numerator and the denominator of the expression above are zero. Hence, in this particular case, it would not be possible to introduce the value that we just encountered in our code. Nevertheless, this difficulty can be overcome at least in two ways:

1. by setting $s=1 / 2$ in $B_{i}, i=1,2,3,4$, before computing these integrals;

2. by computing the limit $s \rightarrow 1 / 2$ in $(\mathrm{A} .3)$.

However, since we already have the expressions of $B_{i}, i=1,2,3,4$, for a general $s$, the second approach is actually straightforward and quicker. Indeed, we can easily compute

$$
\begin{aligned}
\lim _{s \rightarrow \frac{1}{2}} & -h^{1-2 s} \frac{4(k+1)^{3-2 s}+4(k-1)^{3-2 s}-6 k^{3-2 s}-(k+2)^{3-2 s}-(k-2)^{3-2 s}}{2 s(1-2 s)(1-s)(3-2 s)} \\
& =-4(k+1)^{2} \log (k+1)-4(k-1)^{2} \log (k-1)+6 k^{2} \log (k)+(k+2)^{2} \log (k+2)+(k-2)^{2} \log (k-2),
\end{aligned}
$$




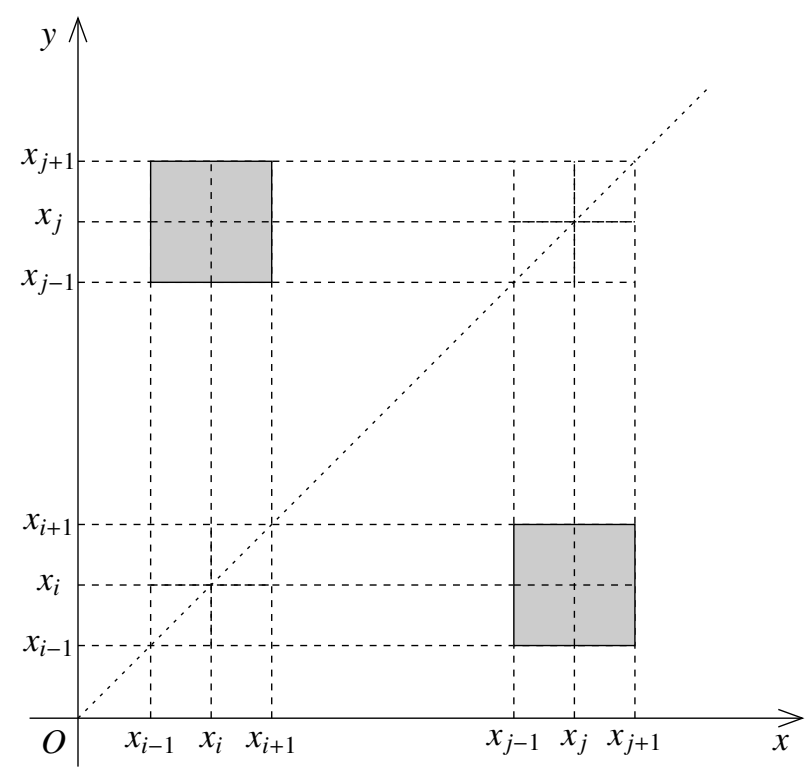

Figure 12: Interactions between the basis function $\phi_{i}$ and $\phi_{j}$ when $j \geq i+2$.

if $k \neq 2$. When $k=2$, instead, since

$$
\lim _{k \rightarrow 2}(k-2)^{2} \log (k-2)=0,
$$

the corresponding value $a_{i, j}=a_{i, i+2}$ is given by $a_{i, i+2}=56 \ln (2)-36 \ln (3)$.

Step 2: $j=i+1$

This is the most cumbersome case, since it is the one with the most interactions between the basis functions (see Figure 5). According to 4.12, and using the symmetry of the integral with respect to the bisector $y=x$, we have

$$
\begin{aligned}
a_{i, i+1}= & \int_{\mathbb{R}} \int_{\mathbb{R}} \frac{\left(\phi_{i}(x)-\phi_{i}(y)\right)\left(\phi_{i+1}(x)-\phi_{i+1}(y)\right)}{|x-y|^{1+2 s}} d x d y \\
= & \int_{x_{i+1}}^{+\infty} \int_{x_{i+1}}^{+\infty} \ldots d x d y+2 \int_{x_{i+1}}^{+\infty} \int_{x_{i}}^{x_{i+1}} \ldots d x d y+2 \int_{x_{i+1}}^{+\infty} \int_{-\infty}^{x_{i}} \ldots d x d y \\
& +\int_{x_{i}}^{x_{i+1}} \int_{x_{i}}^{x_{i+1}} \ldots d x d y+2 \int_{x_{i}}^{x_{i+1}} \int_{-\infty}^{x_{i}} \ldots d x d y+\int_{-\infty}^{x_{i}} \int_{-\infty}^{x_{i}} \ldots d x d y \\
:= & Q_{1}+Q_{2}+Q_{3}+Q_{4}+Q_{5}+Q_{6} .
\end{aligned}
$$

In Figure 13 , we give a scheme of the regions of interactions between the basis functions $\phi_{i}$ and $\phi_{i+1}$ enlightening the domain of integration of the $Q_{i}$. The regions in grey are the ones that produce a contribution to $a_{i, i+1}$, while on the regions in white the integrals will be zero.

Le us now compute the terms $Q_{i}, i=1, \ldots, 6$, separately.

\section{Computation of $Q_{1}$}

Since $\phi_{i}=0$ on the domain of integration we have

$$
Q_{1}=\int_{x_{i+1}}^{+\infty} \int_{x_{i+1}}^{+\infty} \frac{\phi_{i+1}(x)-\phi_{i+1}(y)}{|x-y|^{1+2 s}} d x d y
$$




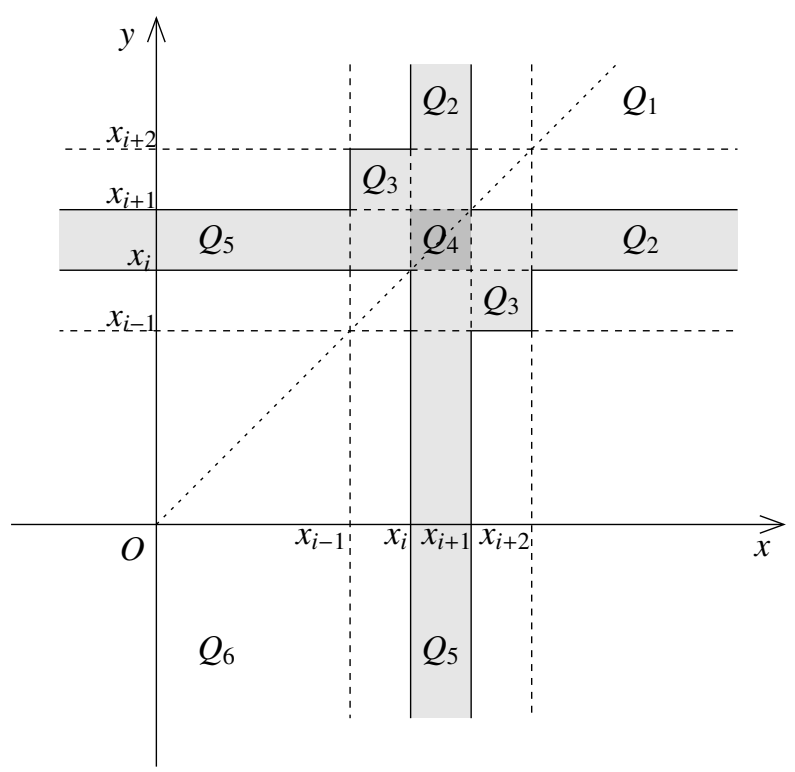

Figure 13: Interactions between the basis function $\phi_{i}$ and $\phi_{i+1}$.

$$
=\int_{x_{i+1}}^{+\infty} \int_{x_{i+1}}^{+\infty} \frac{\phi_{i+1}(x)}{|x-y|^{1+2 s}} d x d y-\int_{x_{i+1}}^{+\infty} \int_{x_{i+1}}^{+\infty} \frac{\phi_{i+1}(y)}{|x-y|^{1+2 s}} d x d y=0 .
$$

The fact that this integral is zero is, actually, not surprising since, according to Figure 13 , the region of integration for $Q_{1}$ is outside of the region of interaction of the basis functions.

\section{Computation of $Q_{2}$}

We have

$$
Q_{2}=2 \int_{x_{i+1}}^{+\infty} \int_{x_{i}}^{x_{i+1}} \frac{\phi_{i}(x)\left(\phi_{i+1}(x)-\phi_{i+1}(y)\right)}{|x-y|^{1+2 s}} d x d y .
$$

Now, using Fubini's theorem we can exchange the order of the integrals, obtaining

$$
\begin{aligned}
Q_{2} & =2 \int_{x_{i}}^{x_{i+1}} \phi_{i}(x) \phi_{i+1}(x)\left(\int_{x_{i+1}}^{+\infty} \frac{d y}{|x-y|^{1+2 s}}\right) d x-2 \int_{x_{i+1}}^{x_{i+2}} \int_{x_{i}}^{x_{i+1}} \frac{\phi_{i}(x) \phi_{i+1}(y)}{|x-y|^{1+2 s}} d x d y \\
& =\frac{1}{s} \int_{x_{i}}^{x_{i+1}} \frac{\phi_{i}(x) \phi_{i+1}(x)}{\left(x_{i+1}-x\right)^{2 s}} d x-2 \int_{x_{i+1}}^{x_{i+2}} \int_{x_{i}}^{x_{i+1}} \frac{\phi_{i}(x) \phi_{i+1}(y)}{|x-y|^{1+2 s}} d x d y \\
& =\frac{1}{s} \int_{x_{i}}^{x_{i+1}} \frac{\left(1-\frac{\left|x-x_{i}\right|}{h}\right)\left(1-\frac{\mid x-x_{i+1}}{h}\right)}{\left(x_{i+1}-x\right)^{2 s}} d x-2 \int_{x_{i+1}}^{x_{i+2}} \int_{x_{i}}^{x_{i+1}} \frac{\left(1-\frac{\left|x-x_{i}\right|}{h}\right)\left(1-\frac{\left|y-x_{i+1}\right|}{h}\right)}{|x-y|^{1+2 s}} d x d y:=Q_{2}^{1}+Q_{2}^{2} .
\end{aligned}
$$

The two integrals above can be computed explicitly. Indeed, employing the change of variables

$$
\frac{x_{i+1}-x}{h}=\hat{x},
$$

and then renaming $\hat{x}=x, Q_{2}^{1}$ becomes

$$
Q_{2}^{1}=\frac{h^{1-2 s}}{s} \int_{0}^{1} x^{1-2 s}(1-x) d x=\frac{h^{1-2 s}}{s(2-2 s)(3-2 s)} .
$$


For computing $Q_{2}^{2}$, instead, we introduce the change of variables

$$
\frac{x_{i}-x}{h}=\hat{x}, \quad \frac{y-x_{i+1}}{h}=\hat{y}
$$

and we obtain

$$
Q_{2}^{2}=-2 h^{1-2 s} \int_{0}^{1} \int_{0}^{1} \frac{(1-x)(1-y)}{(y-x+1)^{1+2 s}} d x d y=h^{1-2 s} \frac{2^{1-2 s}+s-2}{s(1-s)(3-2 s)} .
$$

Adding the two contributions, we get the following expression for the term $Q_{2}$

$$
Q_{2}=h^{1-2 s} \frac{2^{2-2 s}+2 s-3}{s(2-2 s)(3-2 s)} .
$$

\section{Computation of $Q_{3}$}

In this case, we simply take into account the intervals in which the basis functions are supported, so that we obtain

$$
Q_{3}=-2 \int_{x_{i+1}}^{x_{i+2}} \int_{x_{i-1}}^{x_{i}} \frac{\phi_{i}(x) \phi_{i+1}(y)}{|x-y|^{1+2 s}} d x d y=-2 \int_{x_{i+1}}^{x_{i+2}} \int_{x_{i-1}}^{x_{i}} \frac{\left(1-\frac{\left|x-x_{i}\right|}{h}\right)\left(1-\frac{\left|y-x_{i+1}\right|}{h}\right)}{|x-y|^{1+2 s}} d x d y .
$$

This integral can be computed applying again (A.4), and we get

$$
Q_{3}=-2 h^{1-2 s} \int_{0}^{1} \int_{-1}^{0} \frac{(1+x)(1-y)}{(y-x+1)^{1+2 s}} d x d y=h^{1-2 s} \frac{13-5 \cdot 2^{3-2 s}+3^{3-2 s}+s\left(2^{4-2 s}-14\right)+4 s^{2}}{2 s(1-2 s)(1-s)(3-2 s)},
$$

if $s \neq 1 / 2$. If $s=1 / 2$, instead, we have

$$
Q_{3}=-2 \int_{0}^{1} \int_{-1}^{0} \frac{(1+x)(1-y)}{(y-x+1)^{2}} d x d y=1+9 \ln 3-16 \ln (2) .
$$

Notice that this last value could have been computed directly from A.5, by taking the limit as $s \rightarrow 1 / 2$ in that expression, being this limit exactly $1+9 \ln 3-16 \ln (2)$.

\section{Computation of $Q_{4}$}

In this case, we are in the intersection of the supports of $\phi_{i}$ and $\phi_{i+1}$. Therefore, we have

$$
Q_{4}=\int_{x_{i}}^{x_{i+1}} \int_{x_{i}}^{x_{i+1}} \frac{\left(\phi_{i}(x)-\phi_{i}(y)\right)\left(\phi_{i+1}(x)-\phi_{i+1}(y)\right)}{|x-y|^{1+2 s}} d x d y .
$$

Moreover, we notice that, this time, it is possible that $x=y$, meaning that $Q_{4}$ could be a singular integral. To deal with this difficulty, we will exploit the explicit definition of the basis function. We have (see also Figure 14 )

$$
\phi_{i}(x)=1-\frac{x-x_{i}}{h}, \quad \phi_{i+1}(x)=\frac{x_{i+1}-x}{h}, \quad x \in\left(x_{i}, x_{i+1}\right) .
$$

Therefore,

$$
\left(\phi_{i}(x)-\phi_{i}(y)\right)\left(\phi_{i+1}(x)-\phi_{i+1}(y)\right)=\left(\frac{y-x}{h}\right)\left(\frac{x-y}{h}\right)=-\frac{|x-y|^{2}}{h^{2}},
$$

and the integral becomes

$$
Q_{4}=-\int_{x_{i}}^{x_{i+1}} \int_{x_{i}}^{x_{i+1}}|x-y|^{1-2 s} d x d y=-\frac{h^{1-2 s}}{(1-s)(3-2 s)} .
$$




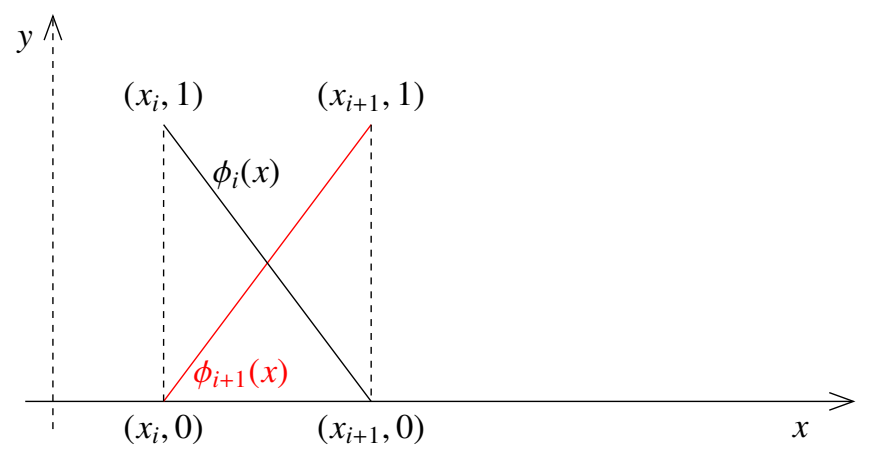

Figure 14: Functions $\phi_{i}(x)$ and $\phi_{i+1}(x)$ on the interval $\left(x_{i}, x_{i+1}\right)$.

\section{Computation of $Q_{5}$}

Here the procedure is analogous to the one for $Q_{2}$ before. Using again Fubini's theorem we have

$$
\begin{aligned}
Q_{5} & =2 \int_{x_{i}}^{x_{i+1}} \phi_{i}(y) \phi_{i+1}(y)\left(\int_{-\infty}^{x_{i}} \frac{d x}{|x-y|^{1+2 s}}\right) d y-2 \int_{x_{i}}^{x_{i+1}} \int_{x_{i-1}}^{x_{i}} \frac{\phi_{i}(x) \phi_{i+1}(y)}{|x-y|^{1+2 s}} d x d y \\
& =\frac{1}{s} \int_{x_{i}}^{x_{i+1}} \frac{\phi_{i}(y) \phi_{i+1}(y)}{\left(y-x_{i}\right)^{2 s}} d y-2 \int_{x_{i}}^{x_{i+1}} \int_{x_{i-1}}^{x_{i}} \frac{\phi_{i}(x) \phi_{i+1}(y)}{|x-y|^{1+2 s}} d x d y .
\end{aligned}
$$

Applying again A.4, it is now easy to check that $Q_{5}=Q_{2}$.

\section{Computation of $Q_{6}$}

In analogy with what we did for $Q_{1}$, we can show that also $Q_{6}=0$.

\section{Conclusion}

The elements $a_{i, i+1}$ are now given by the sum $2 Q_{2}+Q_{3}+Q_{4}$, according to the corresponding values that we computed. In particular, we have

$$
a_{i, i+1}= \begin{cases}h^{1-2 s} \frac{3^{3-2 s}-2^{5-2 s}+7}{2 s(1-2 s)(1-s)(3-2 s)}, & s \neq \frac{1}{2} \\ 9 \ln 3-16 \ln 2, & s=\frac{1}{2} .\end{cases}
$$

Step 3: $j=i$

As a last step, we fill the diagonal of the matrix $\mathcal{A}_{h}$. In this case we have

$$
\begin{aligned}
a_{i, i}= & \int_{\mathbb{R}} \int_{\mathbb{R}} \frac{\left(\phi_{i}(x)-\phi_{i}(y)\right)^{2}}{|x-y|^{1+2 s}} d x d y \\
= & \int_{x_{i+1}}^{+\infty} \int_{x_{i+1}}^{+\infty} \ldots d x d y+2 \int_{x_{i+1}}^{+\infty} \int_{x_{i-1}}^{x_{i+1}} \ldots d x d y+\int_{x_{i+1}}^{+\infty} \int_{-\infty}^{x_{i-1}} \ldots d x d y \\
& +\int_{x_{i-1}}^{x_{i+1}} \int_{x_{i-1}}^{x_{i+1}} \ldots d x d y+2 \int_{-\infty}^{x_{i-1}} \int_{x_{i-1}}^{x_{i+1}} \ldots d x d y++\int_{-\infty}^{+\infty} \int_{x i+1} \ldots d x d y \\
& +\int_{-\infty}^{x_{i-1}} \int_{-\infty}^{x_{i-1}} \ldots d x d y:=R_{1}+R_{2}+R_{3}+R_{4}+R_{5}+R_{6}+R_{7} .
\end{aligned}
$$




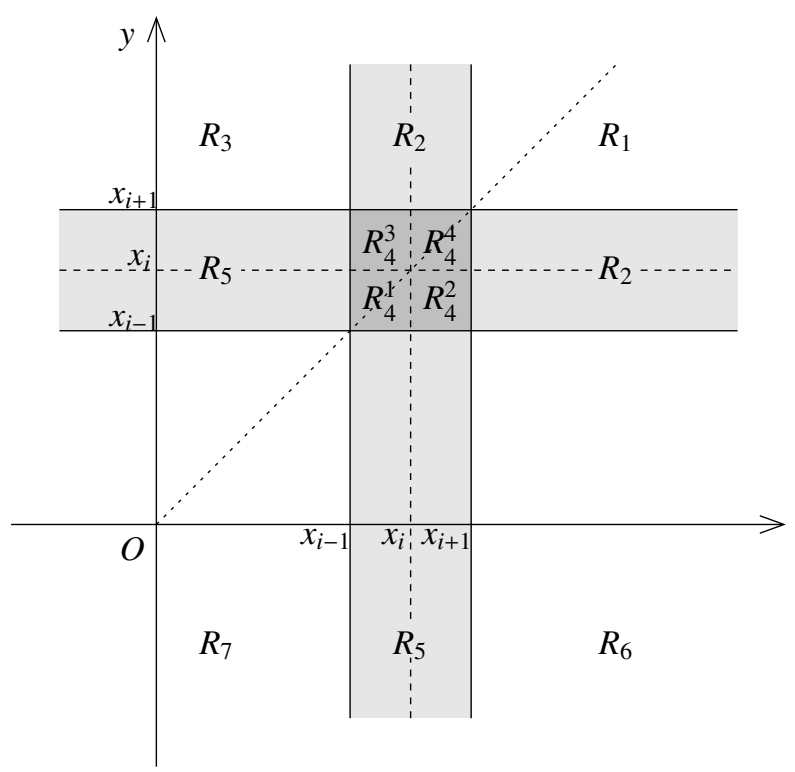

Figure 15: Interactions between the basis function $\phi_{i}(x)$ and $\phi_{i}(y)$.

In Figure 15, we give a scheme of the regions of interactions between the basis functions $\phi_{i}(x)$ and $\phi_{i}(y)$ enlightening the domain of integration of the $R_{i}$. The regions in grey are the ones that produce a contribution to $a_{i, i}$, while on the regions in white the integrals will be zero.

Le us now compute the terms $R_{i}, i=1, \ldots, 7$, separately. First of all, according to Figure 15 we have that $R_{1}=R_{3}=R_{6}=R_{7}=0$. This is due to the fact that the corresponding regions are all away from the support of the basis functions.

\section{Computation of $R_{2}$}

Since $\phi_{i}(y)=0$ on the domain of integrations we have

$$
R_{2}=2 \int_{x_{i+1}}^{+\infty} \int_{x_{i-1}}^{x_{i+1}} \frac{\phi_{i}^{2}(x)}{|x-y|^{1+2 s}} d x d y=2 \int_{x_{i-1}}^{x_{i+1}} \phi_{i}^{2}(x)\left(\int_{x_{i+1}}^{+\infty} \frac{d y}{|x-y|^{1+2 s}}\right) d x=\frac{1}{s} \int_{x_{i-1}}^{x_{i+1}} \frac{\phi_{i}^{2}(x)}{\left(x_{i+1}-x\right)^{2 s}} d x d y .
$$

This integral is computed employing [4.15], and we obtain

$$
R_{2}=\frac{h^{1-2 s}}{s} \int_{-1}^{1} \frac{(1-|x|)^{2}}{(1-x)^{2 s}} d x=h^{1-2 s} \frac{4 s-6+2^{3-2 s}}{s(1-2 s)(1-s)(3-2 s)},
$$

if $s \neq 1 / 2$. If $s=1 / 2$, instead, we have

$$
R_{2}=2 \int_{-1}^{1} \frac{(1-|x|)^{2}}{1-x} d x=2 \ln 16-4
$$

\section{Computation of $R_{4}$}

In this case, we are in the intersection of the supports of $\phi_{i}(x)$ and $\phi_{i}(y)$. Therefore, we have

$$
R_{4}=\int_{x_{i-1}}^{x_{i+1}} \int_{x_{i-1}}^{x_{i+1}} \frac{\left(\phi_{i}(x)-\phi_{i}(y)\right)^{2}}{|x-y|^{1+2 s}} d x d y
$$


In order to compute this integral, we divide it in four components as follows:

$$
\begin{aligned}
R_{4} & =\int_{x_{i-1}}^{x_{i}} \int_{x_{i-1}}^{x_{i}} \ldots d x d y+\int_{x_{i-1}}^{x_{i}} \int_{x_{i}}^{x_{i+1}} \ldots d x d y+\int_{x_{i}}^{x_{i+1}} \int_{x_{i-1}}^{x_{i}} \ldots d x d y+\int_{x_{i}}^{x_{i+1}} \int_{x_{i}}^{x_{i+1}} \ldots d x d y \\
& =R_{4}^{1}+R_{4}^{2}+R_{4}^{3}+R_{4}^{4} .
\end{aligned}
$$

Moreover, we notice that, due to symmetry reason, we have $R_{4}^{2}=R_{4}^{3}$. Therefore, we can compute only one of this two terms and add its value twice when building the matrix $\mathcal{A}_{h}$. Also, notice that in these two region it cannot happen that $x=y$. On the other hand, $R_{4}^{1}$ and $R_{4}^{4}$ may be singular integrals, and we shall deal with them as we did before.

\section{Computation of $R_{4}^{1}$}

Using again the explicit expression of the basis functions we find

$$
\left(\phi_{i}(x)-\phi_{i}(y)\right)^{2}=\frac{|x-y|^{2}}{h^{2}}
$$

and the integral becomes

$$
R_{4}^{1}=\int_{x_{i-1}}^{x_{i}} \int_{x_{i-1}}^{x_{i}}|x-y|^{1-2 s} d x d y=\frac{h^{1-2 s}}{(1-s)(3-2 s)}
$$

\section{Computation of $R_{4}^{2}$}

In this case, we simply have

$$
R_{4}^{2}=\int_{x_{i-1}}^{x_{i}} \int_{x_{i}}^{x_{i+1}} \frac{\left(\phi_{i}(x)-\phi_{i}(y)\right)^{2}}{|x-y|^{1+2 s}} d x d y
$$

Employing 4.15 we obtain

$$
R_{4}^{2}=h^{1-2 s} \int_{-1}^{0} \int_{0}^{1} \frac{(x+y)^{2}}{(x-y)^{1+2 s}} d x d y=h^{1-2 s} \frac{2 s^{2}-5 s+4-2^{2-2 s}}{s(1-2 s)(1-s)(3-2 s)},
$$

if $s \neq 1 / 2$. If $s=1 / 2$, instead, we get

$$
R_{4}^{2}=\int_{-1}^{0} \int_{0}^{1} \frac{(x+y)^{2}}{(x-y)^{2}} d x d y=3-4 \ln 2
$$

\section{Computation of $R_{4}^{4}$}

Also in this case we can use the explicit expression of the basis functions and the integral becomes

$$
R_{4}^{4}=\int_{x_{i}}^{x_{i+1}} \int_{x_{i}}^{x_{i+1}}|x-y|^{1-2 s} d x d y=\frac{h^{1-2 s}}{(1-s)(3-2 s)}=R_{4}^{1} .
$$

Adding the values that we just computed, we therefore obtain

$$
R_{4}=2\left(R_{4}^{1}+R_{4}^{2}\right)= \begin{cases}h^{1-2 s} \frac{8-8 s-2^{3-2 s}}{2 s(1-2 s)(1-s)(3-2 s)}, & s \neq \frac{1}{2} \\ 8 \ln 3-8 \ln 2, & s=\frac{1}{2} .\end{cases}
$$




\section{Computation of $R_{5}$}

Since, once again, $\phi_{i}(y)=0$ on the domain of integration we have

$$
R_{5}=2 \int_{-\infty}^{x_{i-1}} \int_{x_{i-1}}^{x_{i+1}} \frac{\phi_{i}^{2}(x)}{|x-y|^{1+2 s}} d x d y=2 \int_{x_{i-1}}^{x_{i+1}} \phi_{i}^{2}(x)\left(\int_{-\infty}^{x_{i-1}} \frac{d y}{|x-y|^{1+2 s}}\right) d x=\frac{1}{s} \int_{x_{i-1}}^{x_{i+1}} \frac{\phi_{i}^{2}(x)}{\left(x-x_{i-1}\right)^{2 s}} d x d y .
$$

Employing one last time 4.15, we get

$$
R_{5}=\frac{h^{1-2 s}}{s} \int_{-1}^{1} \frac{(1-|x|)^{2}}{(1+x)^{2 s}} d x=h^{1-2 s} \frac{4 s-6+2^{3-2 s}}{s(1-2 s)(3-2 s)(1-s)}=R_{2},
$$

if $s \neq 1 / 2$. If $s=1 / 2$, instead, we have

$$
R_{5}=2 \int_{-1}^{1} \frac{(1-|x|)^{2}}{1+x} d x=8 \ln 2-4
$$

\section{Conclusion}

The elements $a_{i, i}$ are now given by the sum $2 R_{2}+R_{4}$, according to the corresponding values that we computed. In particular, we have

$$
a_{i, i}= \begin{cases}h^{1-2 s} \frac{2^{3-2 s}-4}{s(1-2 s)(1-s)(3-2 s)}, & s \neq \frac{1}{2} \\ 8 \ln 2, & s=\frac{1}{2} .\end{cases}
$$

\section{Funding}

The work of Umberto Biccari was partially supported by the European Research Council [694126 DYCON (Dynamic control and numerics of partial differential equations)], by the Ministerio de Economía, Industria y Competividad (MINECO, Spain) [MTM2014-52347] and by the Air Force Office of Scientific Research [FA9550-15-1-0027].

The work of Víctor Hernández-Santamaría was partially supported by the European Research Council [694126 DYCON (Dynamic control and numerics of partial differential equations)].

\section{Acknowledgements}

The authors wish to acknowledge F. Boyer (Institut de Mathématiques de Toulouse) for fruitful discussion on the numerical implementation of the control problem. Moreover, a special thanks goes to J. Lohéac (Laboratoire de Sciences Numériques de Nantes), for helping with some computations in Section 4.

\section{References}

[1] Acosta, G., Bersetche, F. M., and Borthagaray, J. P. Finite element approximations for fractional evolution problems. arXiv preprint arXiv:1705.09815 (2017).

[2] Acosta, G., Bersetche, F. M., and Borthagaray, J. P. A short FE implementation for a 2d homogeneous Dirichlet problem of a Fractional Laplacian. Comput. Math. Appl. 74, 4 (2017), 784--816.

[3] Acosta, G., and Borthagaray, J. P. A fractional Laplace equation: regularity of solutions and Finite Element approximations. SIAM J. Numer. Anal. 55, 2 (2017), 472--495. 
[4] Adams, R. A., and Fournier, J. J. Sobolev spaces, vol. 140. Academic press, 2003.

[5] Bakunin, O. G. Turbulence and diffusion: scaling versus equations. Springer Science \& Business Media, 2008.

[6] Biccari, U., Warma, M., and Zuazua, E. Local elliptic regularity for the Dirichlet fractional Laplacian. Adv. Nonlinear Stud. 17, 2 (2017), 387--409.

[7] Biccari, U., Warma, M., AND Zuazua, E. Local regularity for fractional heat equations. To appear in SEMA-SIMAI Springer Series, WRAPDE17 (2017).

[8] Bologna, M., Tsallis, C., And Grigolini, P. Anomalous diffusion associated with nonlinear fractional derivative Fokker-Planck-like equation: exact time-dependent solutions. Phys. Rev. E 62, 2 (2000), 2213.

[9] Bonito, A., Lei, W., And Pasciak, J. E. The approximation of parabolic equations involving fractional powers of elliptic operators. J. Comput. Appl. Math. 315 (2017), 32--48.

[10] Bonito, A., ANd Pasciak, J. Numerical approximation of fractional powers of elliptic operators. Mathematics of Computation 84, 295 (2015), 2083--2110.

[11] Borthagaray, J. P. Laplaciano fraccionario: regularidad de soluciones y aproximaciones por elementos finitos. PhD Thesis (2017).

[12] Boyer, F. On the penalised HUM approach and its applications to the numerical approximation of null-controls for parabolic problems. In ESAIM: Proceedings (2013), vol. 41, EDP Sciences, pp. 15--58.

[13] Boyer, F., Hernández-Santamaría, V., and De Teresa, L. Insensitizing controls for a semilinear parabolic equation: a numerical approach. HAL preprint hal-01521642 (2017).

[14] Boyer, F., Hubert, F., And Le Rousseau, J. Uniform controllability properties for space/time-discretized parabolic equations. Numer. Math. 118, 4 (2011), 601--661.

[15] Boyer, F., And Olive, G. Approximate controllability conditions for some linear 1D parabolic systems with space-dependent coefficients. Math. Control Relat. F. 4, 3 (2014), 263--287.

[16] Caffarelli, L., and Silvestre, L. An extension problem related to the fractional Laplacian. Comm. Partial Differential Equations 32, 8 (2007), 1245--1260.

[17] Coron, J.-M. Control and nonlinearity. No. 136. American Mathematical Soc., 2007.

[18] Di Nezza, E., Palatucci, G., and Valdinoci, E. Hitchhiker's guide to the fractional Sobolev spaces. Bull. Sci. Math. 136, 5 (2012), 521--573.

[19] Dipierro, S., Palatucci, G., and Valdinoci, E. Dislocation dynamics in crystals: a macroscopic theory in a fractional Laplace setting. Commun. Math. Phys. 333, 2 (2015), 1061--1105.

[20] Fall, M. M., AND Felli, V. Unique continuation property and local asymptotics of solutions to fractional elliptic equations. Comm. Partial Differential Equations 39, 2 (2014), 354--397.

[21] Fattorini, H. O., And Russell, D. L. Exact controllability theorems for linear parabolic equations in one space dimension. Arch. Rat. Mech. Anal. 43, 4 (1971), 272--292.

[22] Fernández-Real, X., and Ros-Oton, X. Boundary regularity for the fractional heat equation. Rev. R. Acad. Cienc. Exactas Fís. Nat. Ser. A Math. 110, 1 (2016), 49--64.

[23] Fiscella, A., Servadei, R., and Valdinoci, E. Density properties for fractional Sobolev spaces. Ann. Acad. Sci. Fenn. Math 40, 1 (2015), 235--253.

[24] Fursikov, A. V., and Imanuvilov, O. Y. Controllability of evolution equations. Seoul National University, 1996. 
[25] GaL, C. G., AND WARMa, M. Nonlocal transmission problems with fractional diffusion and boundary conditions on non-smooth interfaces. Commun. Partial Differential Equations 42, 4 (2017), 579--625.

[26] Getoor, R. First passage times for symmetric stable processes in space. Trans. Amer. Math. Soc. 101, 1 (1961), 75--90.

[27] Gilboa, G., AND Osher, S. Nonlocal operators with applications to image processing. Multiscale Model. Simul. 7 , 3 (2008), 1005--1028.

[28] Glowinski, R., And Lions, J.-L. Exact and approximate controllability for distributed parameter systems. Acta Numer. 4 (1995), 159--328.

[29] Glowinski, R., Lions, J.-L., AND He, J. Exact and approximate controllability for distributed parameter systems: a numerical approach (Encyclopedia of Mathematics and its Applications). Cambridge University Press, 2008.

[30] Кнорja, F. А., Сhouly, F., and Duprez, M. Partial null controllability of parabolic linear systems. arXiv preprint arXiv:1701.05483 (2017).

[31] Kulczycki, T., Kwaśnicki, M., MaŁecki, J., and Stos, A. Spectral properties of the Cauchy process on half-line and interval. Proc. Lond. Math. Soc. 101, 2 (2010), 589--622.

[32] Kwaśnicki, M. Eigenvalues of the fractional Laplace operator in the interval. J. Funct. Anal. 262, 5 (2012), 2379--2402.

[33] Lebeau, G., and Robbiano, L. Contrôle exact de léquation de la chaleur. Comm. Partial Differential Equations 20, 1-2 (1995), 335--356.

[34] Leonori, T., Peral, I., Primo, A., and Soria, F. Basic estimates for solutions of a class of nonlocal elliptic and parabolic equations. Discrete Contin. Dyn. Syst 35, 12 (2015), 6031--6068.

[35] LeVendorskiI, S. Pricing of the American put under Lévy processes. Int. J. Theor. Appl. Finance 7, 03 (2004), 303--335.

[36] Lions, J.-L., AND Magenes, E. Non-homogeneous boundary value problems and applications I. Springer-Verlag Berlin Heidelberg New York, 1972.

[37] Meerschaert, M. M. Fractional calculus, anomalous diffusion, and probability. Fractional Dynamics (2012), $265--284$.

[38] Micu, S., And Zuazua, E. An introduction to the controllability of partial differential equations. Lecture notes (2004).

[39] Micu, S., and Zuazua, E. On the controllability of a fractional order parabolic equation. SIAM J. Control Optim. 44, 6 (2006), 1950--1972.

[40] MitLeR, L. On the controllability of anomalous diffusions generated by the fractional Laplacian. Math. Control Signals Systems 18, 3 (2006), 260--271.

[41] Nochetto, R. H., OtÁrola, E., ANd Salgado, A. J. A PDE approach to fractional diffusion in general domains: a priori error analysis. Found. Comput. Math. 15, 3 (2015), 733--791.

[42] Рнам, H. Optimal stopping, free boundary, and American option in a jump-diffusion model. Appl. Math. Optim. 35, 2 (1997), 145--164.

[43] Ros-Oton, X., and Serra, J. The Dirichlet problem for the fractional Laplacian: regularity up to the boundary. $J$. Math. Pures et Appl. 101, 3 (2014), 275--302.

[44] Ros-Oton, X., and Serra, J. The extremal solution for the fractional Laplacian. Calc. Var. Partial Differential Equations 50, 3-4 (2014), 723--750. 
[45] Schwartz, L. Etude des sommes d'exponentielles. Hermann, P. Noordhoff Ltd., 1958.

[46] Servadei, R., and Valdinoci, E. On the spectrum of two different fractional operators. Proc. Roy. Soc. Edinburgh Sect. A 144, 04 (2014), 831--855.

[47] Tartar, L. An introduction to Sobolev spaces and interpolation spaces, vol. 3. Springer Science \& Business Media, 2007.

[48] VÁzquez, J. L. Nonlinear diffusion with fractional Laplacian operators. In Nonlinear partial differential equations. Springer, 2012, pp. 271--298.

[49] Zhu, T., ANd Harris, J. M. Modeling acoustic wave propagation in heterogeneous attenuating media using decoupled fractional Laplacians. Geophysics 79, 3 (2014), T105--T116. 